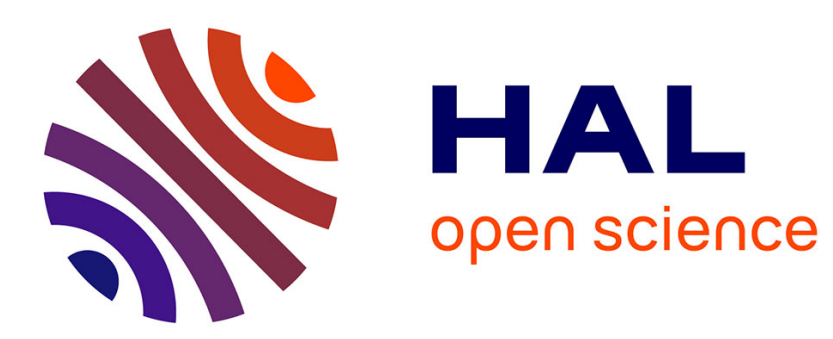

\title{
Quarkonia measurements in heavy-ion collisions in CMS
}

\author{
M. Bedjidian, O. Kodolova
}

\section{To cite this version:}

M. Bedjidian, O. Kodolova. Quarkonia measurements in heavy-ion collisions in CMS. Journal of Physics G: Nuclear and Particle Physics, 2007, 34, pp.N143-N175. 10.1088/0954-3899/34/4/N03 . in2p3-00142648

\section{HAL Id: in2p3-00142648 https://hal.in2p3.fr/in2p3-00142648}

Submitted on 26 Apr 2007

HAL is a multi-disciplinary open access archive for the deposit and dissemination of scientific research documents, whether they are published or not. The documents may come from teaching and research institutions in France or abroad, or from public or private research centers.
L'archive ouverte pluridisciplinaire HAL, est destinée au dépôt et à la diffusion de documents scientifiques de niveau recherche, publiés ou non, émanant des établissements d'enseignement et de recherche français ou étrangers, des laboratoires publics ou privés. 


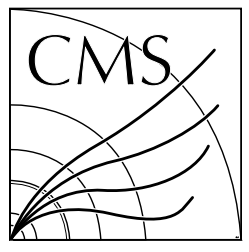

\title{
The Compact Muon Solenoid Experiment

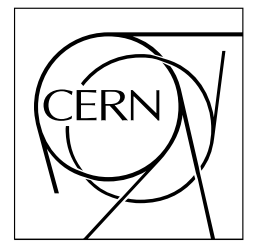

\section{Quarkonia measurements in heavy-ion collisions in CMS}

\author{
M. Bedjidian, \\ IPN, Univ. Claude Bernard Lyon-I and CNRS-IN2P3, France \\ O. Kodolova, \\ Moscow State University, Moscow, Russia
}

\begin{abstract}
The production of quarkonia is one of the most promising signals at the LHC for the study of the production properties of Quark Gluon Plasma. In addition to the $J / \psi$ the extent to which $\Upsilon$ is suppressed should give much insight into the new state of matter. The large muon acceptance and the high precision tracker make the CMS detector ideal for studies of this physics. In this note, the performance of the CMS detector for quarkonia measurements in heavy-ion collisions in the dimuon channel is presented. Dimuon reconstruction efficiencies and mass resolution are calculated using detailed detector simulation. Mass spectra and signal to background ratios are estimated with a fast Monte Carlo program. Results obtained with the fast Monte Carlo are compared with more detailed simulations.
\end{abstract}




\section{Introduction}

The interest in quarkonium production at the LHC emerged from the CERN SPS results $[1,2]$ which showed a strong anomalous suppression of $J / \psi$ production in $\mathrm{Pb}+\mathrm{Pb}$ collisions at $\sqrt{s_{N N}}=17.3 \mathrm{GeV}$. RHIC is studying the $J / \psi$ production in detail at $\sqrt{s_{N N}}=200 \mathrm{GeV}$ in $\mathrm{Au}+\mathrm{Au}$ and $\mathrm{Cu}+\mathrm{Cu}$ collisions. The global $\mathrm{J} / \psi$ suppression ammounts in factor three between most central and peripheral collisions [3]. Only part of the suppression can be explained by calculation including shadowing and absorption effects [4]. However recent theoretical analysis [5] suggests that the direct $J / \psi$ could survive for temperature as high as $1.5 \mathrm{Tc}$ (The critical temperature for the phase transition is about $200 \mathrm{MeV}$ ) which could be out of the range of the RHIC.

Although the $\Upsilon$ production cross section is large enough to be observed at RHIC, albeit with limited statistics, its suppression is not expected until the high initial temperatures foreseen at LHC are reached.

Thanks to its large muon detector and to the high precision tracker, the CMS detector is particularly well suited to study the quarkonia state production in the dimuon channel.

Over than 4 orders of magnitude separate the $\mathrm{Pb}+\mathrm{Pb}$ inelastic cross section from the production cross section of the Upsilon in the dimuon decay channel. Therefore, a complete and statistically significant simulation of the quarkonia detection using an event generator like HIJING [6] to simulate a $\mathrm{Pb}+\mathrm{Pb}$ collision and the official tools of the CMS Collaboration for the tracking of the secondaries, OSCAR [7], together with the reconstruction of the event, ORCA [8], would need an unavailable calculation power.

A fast simulation method is therefore unavoidable to study the $\Upsilon$ and $J / \psi$ production in the heavy ion collisions.

The fast Monte Carlo method is described and compared with the result of the detailed simulation. A full detector and trigger simulation simulation plus reconstruction are carried out for a few $10^{7}$ particles of different types relevant to the muon signal and background (muons, pions, kaons, $b-, c-$ hadrons. The corresponding response functions (trigger acceptances, mass resolutions, reconstruction efficiencies, etc) are parametrized with detailed simulations for the each type of particle. The obtained parametrizations are used in a fast MC to produce the finally corrected yields.

The performances of CMS for $\Upsilon$ and $J / \psi$ observation are discussed.

\section{CMS detector}

A detailed description of the detector elements can be found in the corresponding Technical Design Reports [9, 10, $11,12]$.

The CMS detector is designed to identify and measure muons, electrons, photons and jets over a large energy and rapidity range. It offers the widest muon acceptance centered at midrapidity. CMS is particularly well suited to study the $\Upsilon$ and $J / \psi$ families, the continuum up to the $Z^{0}$ mass. The CMS dilepton capability allows systematic studies of heavy flavour physics. The impact parameter (centrality) of the collision can be determined from measurements of transverse energy production over the range $|\eta|<5$.

The central element of CMS is the magnet, a $13 \mathrm{~m}$ long, $6 \mathrm{~m}$ diameter, high-field solenoid (a uniform $4 \mathrm{~T}$ field) with an internal radius of $\approx 3 \mathrm{~m}$. The hadronic (HCAL) and electromagnetic (ECAL) calorimeters are located inside the coil (except the forward calorimeter) and cover (including the forward calorimeter) from -5 to 5 pseudorapidity units. The HF calorimeter covers the region $3<|\eta|<5$. The ECAL calorimeter consists from the $20 \mathrm{~cm}$ length $\mathrm{PbWO} 4$ crystalls and corresponds to 1.1 interaction length. The central hadron calorimeter $(|\eta|<3)$ is a sampling calorimeter: it consists of active material inserted between brass alloy absorber plates. Innemost and outermost absorbers are produced from stainless steel. The overall number of the nuclear interaction length before penetrating muon stations $(\lambda)$ is $11-16$ over $|\eta|<3$ [13]. Muons loose $3 \mathrm{GeV}$ in the calorimeter due-to ionization losses. The probablity for hadron to not interact in calorimeter at al is $0.005 \%$. The first absorber, the electromagnetic calorimeter, is $1.3 \mathrm{~m}$ from the interaction point, eliminating a large fraction of the hadronic background.

The tracker and muon chambers cover the pseudorapidity region $|\eta|<2.4$,

Starting from the beam axis the tracker is composed of two different types of detectors: pixels and silicon strips. The pixel detector consists of 3 barrel layers located at $4,7,11 \mathrm{~cm}$ from the beam axis with granularity $150 \times 150 \mu \mathrm{m}^{2}$ and 2 forward layers with granularity $150 \times 300 \mu \mathrm{m}^{2}$ located at the distances of 34 and $43 \mathrm{~cm}$ in $z$ from the center of detector.

Silicon strip detectors are divided into inner and outer sections and fill the tracker area from $20 \mathrm{~cm}$ to $110 \mathrm{~cm}(10$ layers) in the transverse direction and up to $260 \mathrm{~cm}$ (12 layers) in longitudinal direction. The strip length for silicon 
strip counters varies up to $21 \mathrm{~cm}$ for outermost layers and the pitch varies from 61 to $205 \mu \mathrm{m}$ depending on the radius. The $\eta$-coverage of tracker detector is from -2.4 to 2.4 .

The CMS muon stations consist of drift tube chambers (DT) in the barrel region (MB), $|\eta|<1.2$, cathode strip chambers (CSCs) in the endcap regions (ME), $0.9<|\eta|<2.4$, and resistive plate chambers (RPCs) in both barrel and endcaps, for $|e t a|<1.6$. The chambers are mounted within iron wheels in barrel and iron disks in endcap. The RPC detector is dedicated to triggering, while the DT and CSC detectors, used for precise momentum measurements, also have the capability to self-trigger up to $|e t a|<2.1$. The muon system can thus reconstruct muons in the range $|e t a|<2.4$.

\section{Input to Monte Carlo studies}

At the LHC energies a possible regeneration of the J/Psi, due to the high production of c-bar c pairs, is expected and may compensate an anomalous suppression [14]. Therefore, in the work presented here, the shadowing is the only effect taken into account. Dense matter effects like suppression or modification in the high Pt hadron spectra [25] are not considered.

\subsection{Quarkonia production rates}

The quarkonium cross sections per nucleon in $\mathrm{Pb}+\mathrm{Pb}$ interactions are calculated in the color evaporation model [16, $18,19]$. These are NLO calculations. The parameters (PDF functions and scales) and the model prescription are indicated in [16] at page 258-259. The calculations include nuclear shadowing, but no additional effect like the absorption is taken into account, since the interplay of shadowing and nucleon absorption as a function of $\sqrt{s}$ is not known.

Table 1 shows the inclusive quarkonia production cross section values in the case of $\mathrm{Pb}+\mathrm{Pb}$ collisions. The values are averaged over impact parameter. The dependence of the resonance cross-sections and/or kinematical spectra on the impact parameter is not considered.

Table 1: Inclusive cross sections for quarkonium production in minimum bias $\mathrm{Pb}+\mathrm{Pb}$ collisions multiplying by decay branching ratio into $\mu^{+} \mu^{-}$.

\begin{tabular}{|c|c|c|c|c|}
\hline \multicolumn{5}{|c|}{$B_{\mu \mu} \sigma_{\text {prod }}(\mu \mathrm{b})$} \\
\hline$J / \psi$ & $\psi^{\prime}$ & $\Upsilon$ & $\Upsilon^{\prime}$ & $\Upsilon^{\prime \prime}$ \\
\hline 48930 & 879 & 304 & 78.8 & 44.4 \\
\hline
\end{tabular}

The kinematical distributions in $p_{\mathrm{T}}$ and $\eta$ of the quarkonia (the pseudorapidity is more convenient to indicate the $\eta$ coverages of the different CMS detector regions under consideration), according to [16] are shown in Figs. 1, 2. We can note here that the transverse momentum distribution of $\Upsilon$ have a much longer tail than the one of $J / \psi$. The same $p_{\mathrm{T}}$ and $\eta$ distributions are used for the different states in the same quarkonium family, i.e. all $\Upsilon$ states are generated with $\Upsilon$ kinematical distributions and the $\psi^{\prime}$ is generated with the same kinematical distributions of the $J / \psi$.

\subsection{Muon background rates}

The choice of the impact parameter, $b$, governs the overall charged particles multiplicity, $N_{c h}$, as well as the number of open heavy quark pairs, $\mathrm{N}_{Q \bar{Q}}$ produced in the collision. Charged particles and open charm and bottom production give the essential contributions to the dimuon background. 

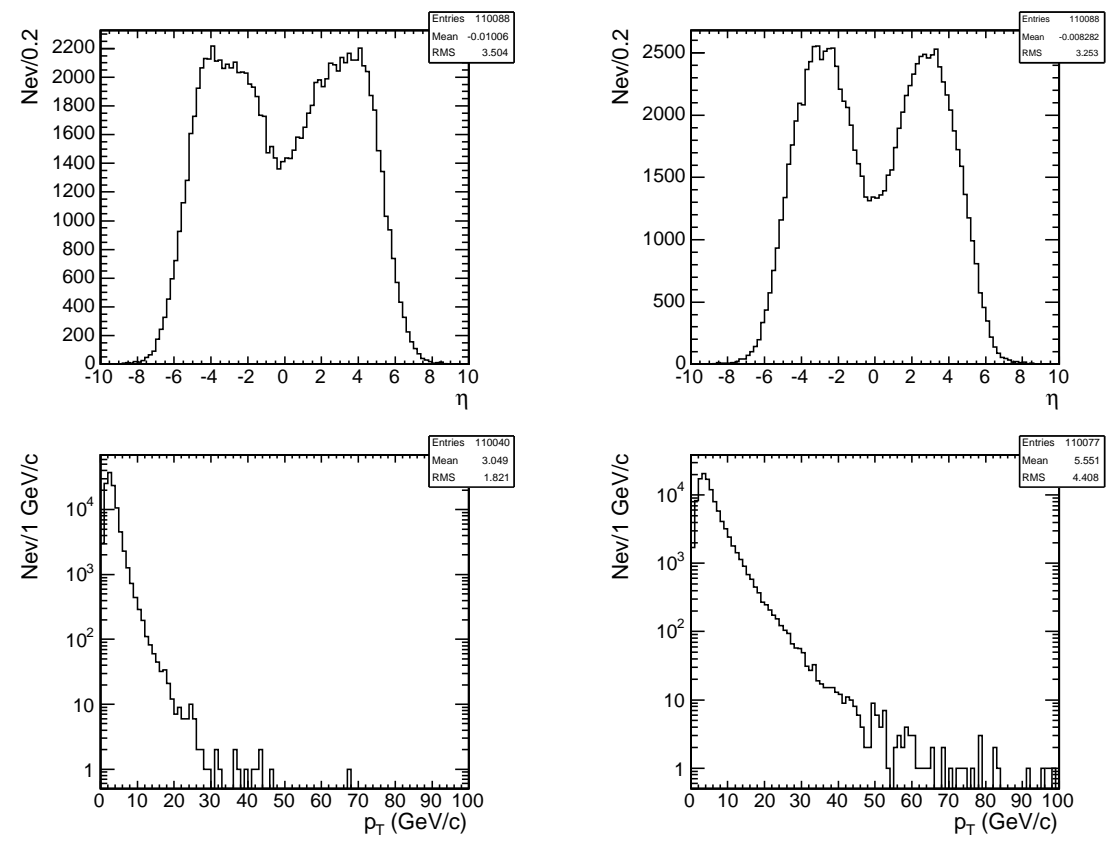

Figure 1: Kinematical distributions, $p_{\mathrm{T}}$ and $\eta$ of the quarkonia, $J / \psi$ on the left and $\Upsilon$ on the right, at the generation level [16].
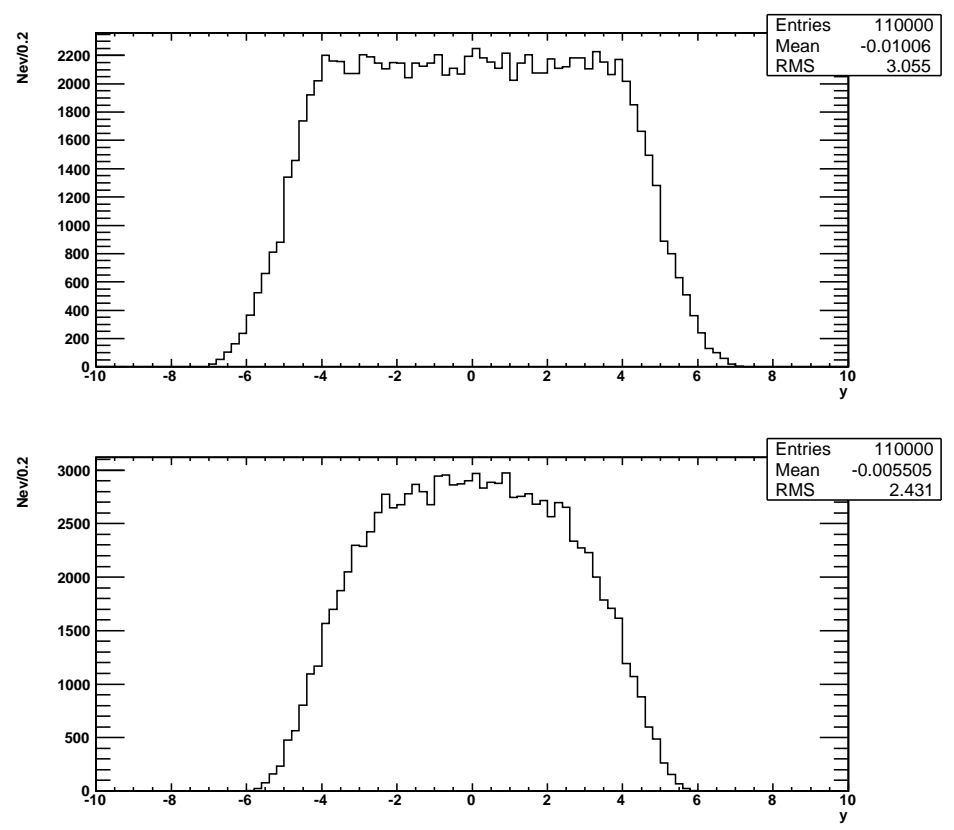

Figure 2: The rapidity distribution of the quarkonia, $J / \psi$ at the top and $\Upsilon$ at the bottom, at the generation level [16].

\subsubsection{From pions and kaons}

The soft hadrons multiplicity at impact parameter $b=0$ is adjusted to the value expected at the LHC. Although extrapolations from RHIC suggest $d N^{ \pm} /\left.d \eta\right|_{\eta=0}$ as low as 2000 [20], two hypotheses with higher multiplicity values are used:

a) high multiplicity value with $\mathrm{d} N^{ \pm} / \mathrm{d} \eta=5000$ at $\eta=0$

b) low multiplicity with $\mathrm{d} N^{ \pm} / \mathrm{d} \eta=2500$

In the fast Monte Carlo, only pions and kaons, which all together represent about $90 \%$ of all charged particles emitted in the collision, are considered. Neither neutral hadrons nor gammas are taken into account. 
Protons can be registered as muons in muon chambers either due-to punchthrough of hadronic shower in the CMS calorimeter (11-16 $\lambda$ in barrel and $11 \lambda$ in endcap) $[13,22]$ or via the direct penetration of surviving protons into muon stations. The punchthrough in the muon chambers due-to hadronic shower in the CMS calorimeter is less then $1.2 \times 10^{-5}$ for hadrons with $p_{T}<10 \mathrm{GeV} / \mathrm{c}$ [21]. The probability of the direct penetration of the surviving proton into muon stations is lower than $1.6 \times 10^{-5}$.

For pions and kaons, the overall probability (punchthrough and decay into muon) to be registered is about 0.003 and 0.01 correspondingly (Sect. 3.3) and is more than two order of magnitude higher then the probability to register proton. Therefore, protons are neglected in the present study.

Kaons and pions are distributed according a ratio $\mathrm{K} / \pi=11 \%$ given by HIJING. In order to be conservative, these two sets of multiplicity are not considered $a t b \approx 0$ but for the $5 \%$ most central collisions. For the high multiplicity set, Table 2 shows the number of pions and kaons in three cases: at $\mathrm{b} \approx 0$, for the $5 \%$ most central $\mathrm{Pb}+\mathrm{Pb}$ collisions and in the case of minimum bias. Switching to the low multiplicity set divides the numbers for $\mathrm{d} N^{ \pm} / \mathrm{d} \eta$ by two.

The transverse momentum and the pseudorapidity of the particle are chosen randomly from distributions extracted from HIJING. Pions and kaons have quite different $p_{\mathrm{T}}$ distributions: the mean $p_{\mathrm{T}}$ value for pions is $0.44 \mathrm{GeV} / c$ while for kaons it is equal to $0.6 \mathrm{GeV} / c$ This fact explains why in spite of the $\mathrm{K} / \pi$ ratio of $11 \%$ at the generation level this ratio grows up to $80 \%$ at the detector level.

Table 2: Main physical parameters for $\mathrm{PbPb}$ collisions with high multiplicity, used in the fast MC analysis.

\begin{tabular}{|r|c|c|c|}
\cline { 2 - 4 } \multicolumn{1}{c|}{} & $\mathrm{b} \approx 0 \mathrm{fm}$ & $5 \%$ c.c & min. bias \\
\hline $\mathrm{d} N^{ \pm} / \mathrm{d} \eta \pi^{ \pm}+K^{ \pm}$ & 4980 & 4600 & 1380 \\
\hline $\mathrm{N}(c \bar{c})$ w/o shadowing & 226 & 206 & 51 \\
w/ shadowing & 150 & 135 & 33 \\
\hline $\mathrm{N}(b \bar{b})$ w/o shadowing & 6 & 5.5 & 1.4 \\
w/ shadowing & 5 & 4.7 & 1.2 \\
\hline
\end{tabular}

\subsubsection{From open heavy quark pair production}

The other important source of background are the muons coming from the open $Q \bar{Q}$ pair production produced in individual $\mathrm{NN}$ collisions. The number of pairs produced in $\mathrm{Pb}+\mathrm{Pb}$ collisions as a function of impact parameter $b$ is

$$
N(Q \bar{Q})=\sigma(Q \bar{Q}) T_{A A}(b)
$$

directly proportional to the nuclear overlap $T_{A A}(b)$ where $T_{\mathrm{PbPb}}(0)=30.4 / \mathrm{mb}$. The NLO $Q \bar{Q}$ production cross sections in $5.5 \mathrm{TeV} p p$ interactions are $\sigma(c \bar{c})=7.5 \mathrm{mb}$ and $\sigma(b \bar{b})=0.2 \mathrm{mb}$. These cross sections do not include the shadowing effect, the reducing factor of which is taken to be $35 \%$ and $15 \%$ respectively [23]. The number of $Q \bar{Q}$ pairs at $b=0$ for $\mathrm{Pb}+\mathrm{Pb}$ collisions, without and with the shadowing effect, are shown in Table 2 . These numbers are assumed to be the same for both sets of $\pi / \mathrm{K}$ multiplicity.

Each $Q \bar{Q}$ has a probability to give one or more muons in the final state. These probabilities for $c \bar{c}$ are $0.819,0.171$, 0.010 for zero, one and more then two muons correspondingly. For $b \bar{b}$ the corresponding vaues are $0.626,0.309$, 0.065. They were obtained from Pythia 6.205 event generator. The muon $p_{\mathrm{T}}$ and $\eta$ distributions are extracted from [16] (Fig. 3). The large difference in the average $p_{\mathrm{T}}$ values $\left\langle p_{\mathrm{T}}^{\mu}\right\rangle_{c}=0.55 \mathrm{GeV} / c$ and $\left\langle p_{\mathrm{T}}^{\mu}\right\rangle_{b}=1.45 \mathrm{GeV} / c$ counterbalances the effect of the much larger number of $c \bar{c}$ pairs at the generation level. 

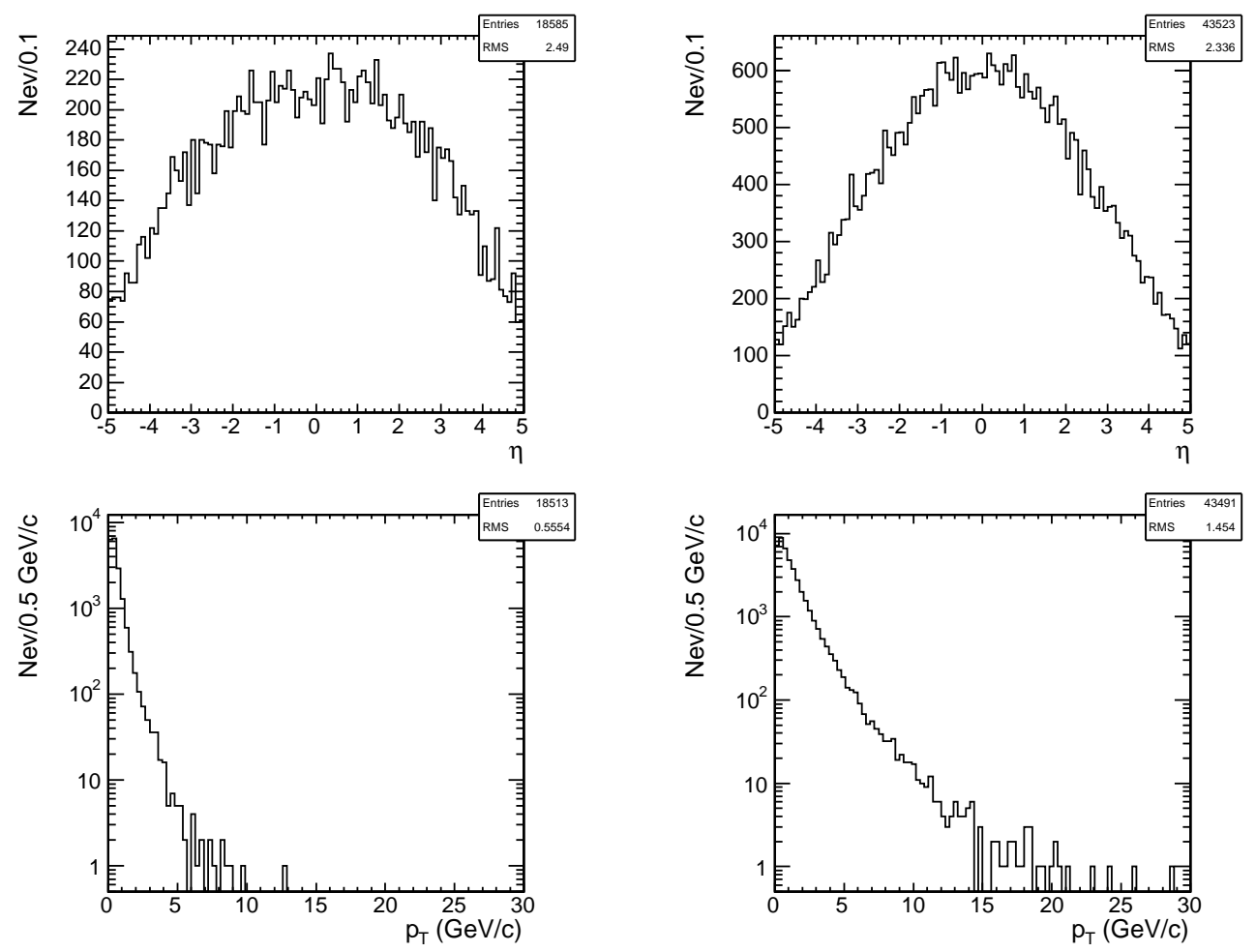

Figure 3: $p_{\mathrm{T}}$ and $\eta$ distributions at the generation level of the muons from $Q \bar{Q}$ pair production. $c \bar{c}$ on the left and $b \bar{b}$ on the right [16].

\subsection{Detector response}

The $\mathrm{Pb}-\mathrm{Pb}$ event produces pions and kaons, which decay far from the vertex and $\mathrm{b}$ - and $\mathrm{c}$ - mesons, decaying a few $\mathrm{mm}$ far away from the primary vertex. At the vertex level it is not known whether a pion/kaon will decay or will have a nuclear interaction in the calorimeters. In very rare cases it may also reach a muon station. Hadrons can also give punch-through and be registered. A set of tables with the probability of particles of different types, produced in the primary vertex, to be identified by the detector as a "muons", are produced. The particles in event are combined by pairs and each pair is weighted according to its probability of being registered. The weighting procedure is described in details in sections 4.1, while the reconstruction errors for weights are presented in section 5.4 .

\subsubsection{Muon trigger tables}

The response of CMS detector to the incident pions, kaons and muons is given under the form of acceptance and trigger tables. They are 2-dimensional tables in $\mathrm{P}, \eta$ space. Incident particles with both signs are generated at the center of the detector with a given set of $\mathrm{P}, \eta$ values. Finally, the particle is accepted or rejected according to criteria specific to the heavy ion dimuon trigger (Sect.3.3.2). 

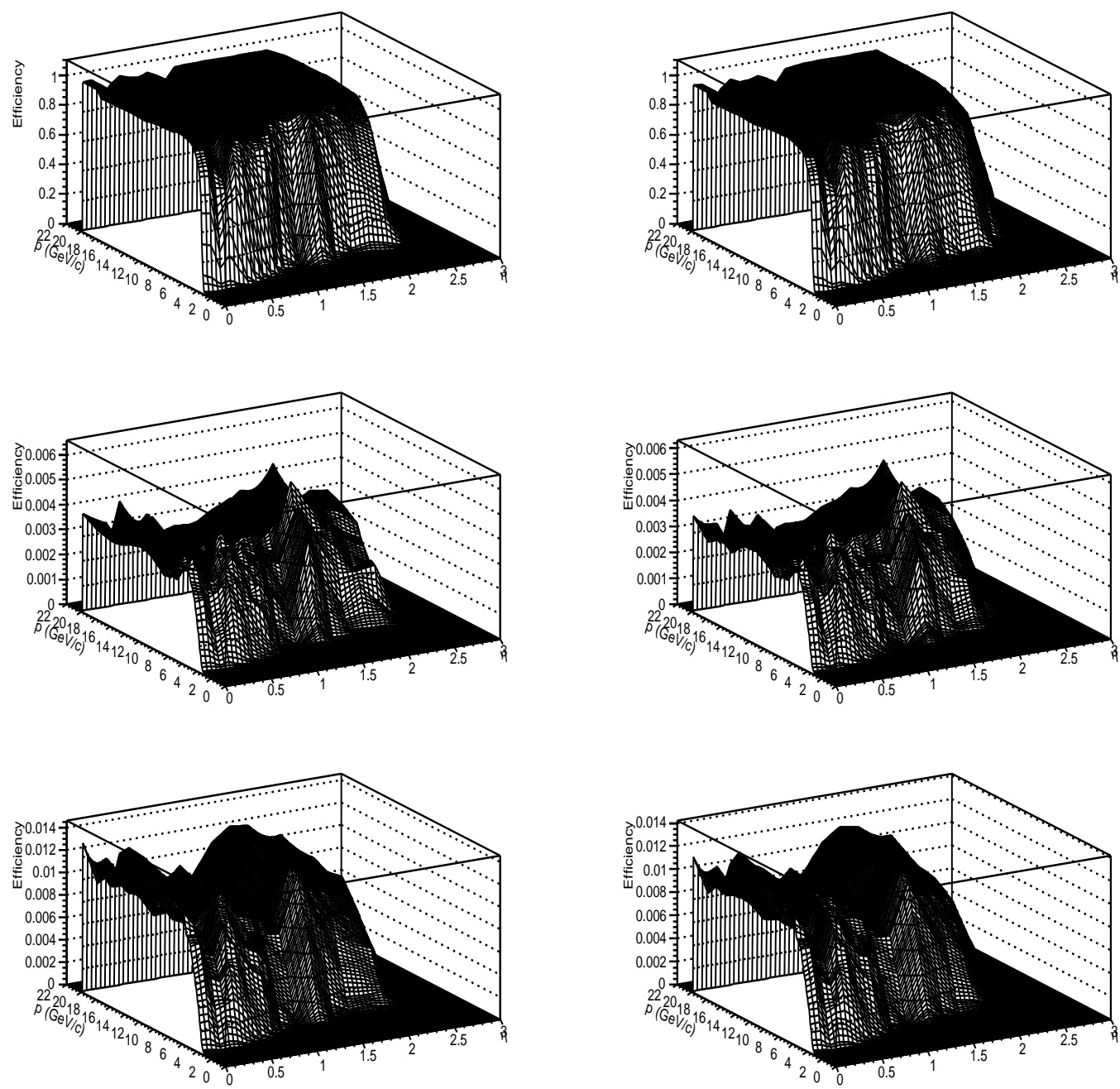

Figure 4: Acceptance and trigger tables for muons (up), pions (middle) and kaons (bottom) for level I (left) and level II (right) heavy ion dimuon trigger in dependence on the momentum $(\mathrm{p})$ and pseudorapidity $(\eta)$ of the particles at the primary vertex

To minimize computing time, particles were generated in a limited number of points in the $\mathrm{P}, \eta$ space.

27 points along $\mathrm{P}$ and 19 points in $\eta$ dimension.

While for the accepted muons the statistics is high enough (for 6000 incident muons efficiencies above $90 \%$ could be reached in most of $\mathrm{P}, \eta$ points) the situation is much less favourable for the hadrons for which the efficiency is below $1 \%$. Nevertheless the number of generated mesons stays between 20000 and 160000 .

Two tables are built at once. They correspond to Level I and Level II trigger. The final trigger tables are done with a binning of $0.1 \mathrm{GeV} / c$ in $\mathrm{P}$ and 0.05 in $\eta$. The intermediate points are linearly interpolated from the neighbours. The final tables are shown in Fig. 4. Each channel content is the trigger efficiency $\epsilon_{t r i g}^{L V L 1}$ and $\epsilon_{t r i g}^{L V L 2}$, respectively.

\subsubsection{Track reconstruction}

\subsubsection{Dimuon reconstruction algorithm}

Registration of dimuons starts with single L1 muon trigger with requirement of low quality muon candidates [13]. The baseline of the default heavy ion L1 trigger selection described in L1 Trigger TDR [13] is based on a single muon with a $p_{T}$ cut ranging from about $3.5-4 \mathrm{GeV}$ in the barrel to about $2-1.5 \mathrm{GeV}$ in the endcap up to $\eta$ of 2.4. This is achieved through an OR of the DT, CSC and RPC. The possibility to choose the logic with a different chamber combination is foreseen. The events accepted by the L1 trigger are passed through the L2 trigger. At this stage, the criteria for dimuons is either two opposite sign muons at L1 or two opposite sign muons at L2. The selection efficiency for the $J / \psi$ is $1 \%$ and the selection efficiency of $\Upsilon$ is $20 \%$. The selection efficiency of the 
HIJING events after L1 and L2 trigger selection is 1.7\% and is discussed in Sect.5.5.1.

As soon as the like sign pairs are also needed, they can be selected with the symmetric condition: either two like sign muons at L1 or two like sign muons at L2.

After successive L1/L2 trigger conditions, each of the muon candidates is propagated within the tracker volume with look-up tables (fast muon propagator) $\left|\phi_{L 1 / L 2}-\phi_{\mu}\right|$ vs $P_{T}^{L 1 / L 2}$ in barrel muon stations or $\left|\phi_{L 1 / L 2}-\phi_{\mu}\right|$ vs $P_{Z}^{L 1 / L 2}$ in endcap muon stations. These look-up tables are constructed with the prompt muon samples from upsilon and $\mathbf{J} \psi$ decays. Inputs to the fast muon propagator are the momentum and position of the muon candidates in the muon chambers from the L1 or L2 trigger response. The fast muon propagator gives as outputs the position and the direction of the track at the outer tracker layer. The absolute value of the momentum is assigned as it is determined by the L1 or L2 trigger. Each reconstructed hit (rechit), found inside the wide window, is considered as the muon seed with the momentum determined by the muon stations. The width of the window is the parameter of the algorithm and can be made wider or narrower depending on the occupancy at the outer tracker layer. The initial aperture of the window corresponds to three-five sigmas of the multiple scattering angle within the calorimeter material for the muons with a momentum of 3-4 GeV/c. The further narrowing window depends on the ratio between reconstruction efficiency and purity. During data taking the dependence of the window size on the multiplicity has to be determined for each range of the occupancy level in the tracker, assuming that the model describes perfectly the detector. The update of the momentum of the seed is done with the look-up tables $\left|\phi_{L 1 / L 2}-\phi_{\text {rechit }}\right|$ vs $P_{T}^{g e n}$ in the tracker part that corresponds to muon barrel stations $(|\eta|<1.2)$ and $\left|\phi_{L 1 / L 2}-\phi_{\text {rechit }}\right|$ vs $P_{Z}^{g e n}$ in the tracker part that corresponds to muon endcap stations $(|\eta|>1.2)$. These tables are created with the same prompt muon samples from upsilon and $\mathbf{J} \psi$ decays. The reconstructed hits which provide $\left|\left(P_{T}^{g e n}-P_{T}^{L 1 / L 2}\right) / P_{T}^{g e n}\right|>$ cut or $\left|\left(P_{Z}^{g e n}-P_{Z}^{L 1 / L 2}\right) / P_{Z}^{g e n}\right|>$ cut are removed from the list of seeds.

Pattern recognition and track reconstruction inside the tracker volume determines to a large extent the feasibility of heavy ion physics in CMS [24]. The algorithm is the modification of the regional track finder and is based on the muons seeded by the muon stations and on the knowledge of the primary vertex. With these two restrictions, the different types of tracks seeding, tracks propagation and update of track trajectory inside the tracker volume can be used. Current study is based on the algorithm developed in [24].

The further propagation of the track inside the tracker volume is performed towards the primary vertex with GtfPropagator ([13]). The update of the track parameters during the propagation through the successive layers is performed with a simple parametrization done in the cylindrical coordinates $(\mathrm{r}, \phi, \mathrm{z})$. The parametrization is separated in the transverse and longitudinal planes and depends on whether the track intersects the barrel or the forward layers:

a) barrel cylinders

The difference $\delta \phi(i, j)$ between the azimuthal angles of the two clusters $(\mathrm{i}, \mathrm{j})$ is calculated from the expression:

$$
\delta \phi(i, j)=\arcsin \left(r_{i} /(2 R)\right)-\arcsin \left(r_{j} /(2 R)\right) \sim K \frac{\delta r_{i, j}}{P_{T}}
$$

where $\mathrm{R}$ is the radius of the muon trajectory and $r_{i}, r_{j}$ are the radii of the two reconstructed hits located on the different detector layers, $\delta r_{i, j}=\left|r_{i}-r_{j}\right|$ is the difference of the radii of the reconstructed hits.

b) Forward disks

In the forward tracker $\phi$ and $z$ are well measured and a muon track can be parametrized as follows:

$$
\delta \phi(i, j)=\frac{K \delta z_{i, j}}{P_{L}}
$$

The parametrization in the longitudinal plane is considered linear and is used to determine roughly the pseudorapidity of the track. During propagation from layer to layer, the cuts on the difference between the predicted position of track and reconstructed hits are applied.

As soon as a set of possible muon candidates is selected, they are fit with Kalman smoother [13]. The pair of opposite sign tracks are collected and fit, pair by pair, with the principal vertex finder [13]. The cuts on $\chi^{2}$ of the tracks and vertex are applied.

All cuts are the parameters of the algorithm and depend on the layer occupancy. The combination of cuts has to be determined for the different occupancy levels assuming the model of the detector to be perfect. 
For the first propagation, tracks are assumed to intersect all successive layers without any gaps. If the vertex is failed, the propagation starts again allowing one gap for each track. The layers are considered to be missed one by one and with each configuration of the layers, the vertex existence is checked. All successful tracks are propagated back to the muon stations [13] either to the cylinder with radius equal to the radius of the first muon track point in the barrel or to a Z-coordinate corresponding to the Z-coordinate of the first muon track point in the endcap. The first muon track point is given either by the level one or by the level two muon trigger. The cut on the difference between muon track coordinates reconstructed by the trigger and predicted by the propagation is determined with the muon sample.

The reconstruction can be done both in the trigger logic and in the off-line reconstruction logic:

- trigger logic As soon as a vertex is successfully found, the cycle on the missed layers is stopped and the event is considered as successful. The condition is to find at least one dimuon vertex.

- off-line reconstruction logic The treatment of the missed layers is done in the same manner. But the algorithm is stopped only when the possibility of the vertex reconstruction is checked for each pair of opposite sign muon triggers. The condition is to find all possible dimuon vertices.

Similarly, the like-sign muon pairs can be reconstructed in the same manner.

\subsubsection{Dependence of the reconstruction efficiency on the particle type}

The pseudorapidity range $|\eta|<2.4$ is divided into 4 bins: $|\eta|<0.7 ; 0.7<|\eta|<1.2 ; 1.2<|\eta|<2.0 ; 2.0<|\eta|<2.4$. The momentum of the particles is divided in 4 bins: $3 \mathrm{GeV} / \mathrm{c}<p<5 \mathrm{GeV} / \mathrm{c} ; 5 \mathrm{GeV} / \mathrm{c}<p<10 \mathrm{GeV} / \mathrm{c} ; 10$ $\mathrm{GeV} / \mathrm{c}<p<15 \mathrm{GeV} / \mathrm{c} ; 15 \mathrm{GeV} / \mathrm{c}<p<20 \mathrm{GeV} / \mathrm{c}$. Inside a $(\mathrm{p}, \eta)$ cell, the distributions are taken as uniform. A pair of particles is generated in each cell. For example: if both particles have momentum $3<p<5$ then they are both generated with $|\eta|<0.7$ or $0.7<|\eta|<1.2$ or $1.2<|\eta|<2.0$ or $2.0<|\eta|<2.4$. In addition, pairs with one particle in one pseudorapidity bin and the other in an another pseudorapidity bin were generated as a cross-check. The same $|\eta|$ division is done for the other three momentum bins.

The dependence of the efficiency on the particle momenta is shown in Figs 5 for the different particle types with both particles at $|\eta|<0.7$.

Figures 6-9 show the dependence of the tracking efficiency on the pseudorapidity for different particle types and different momentum regions.

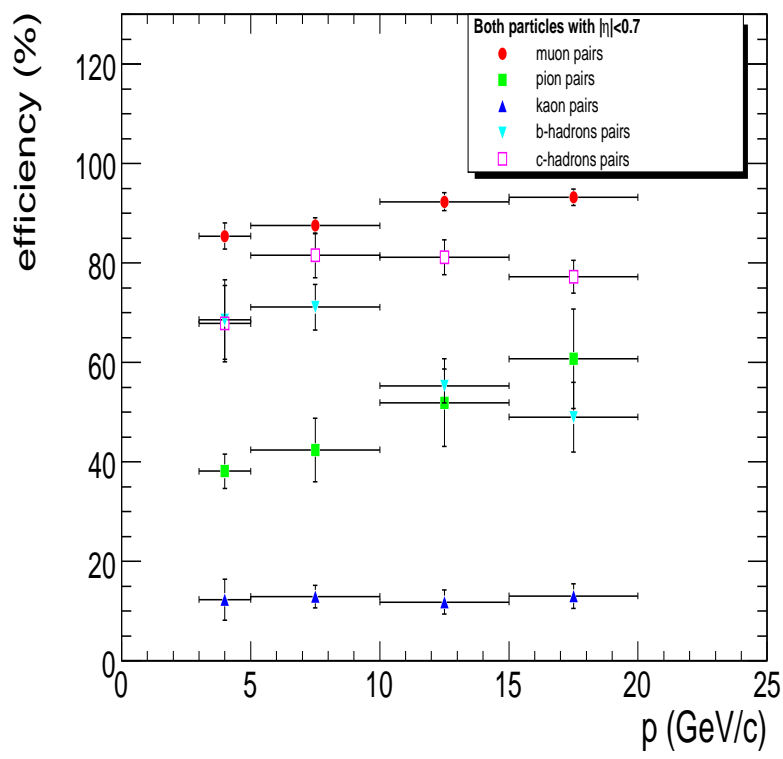

Figure 5: The dependence of the tracking efficiency on the momentum for pairs with both particles in $|\eta|<0.7$ 


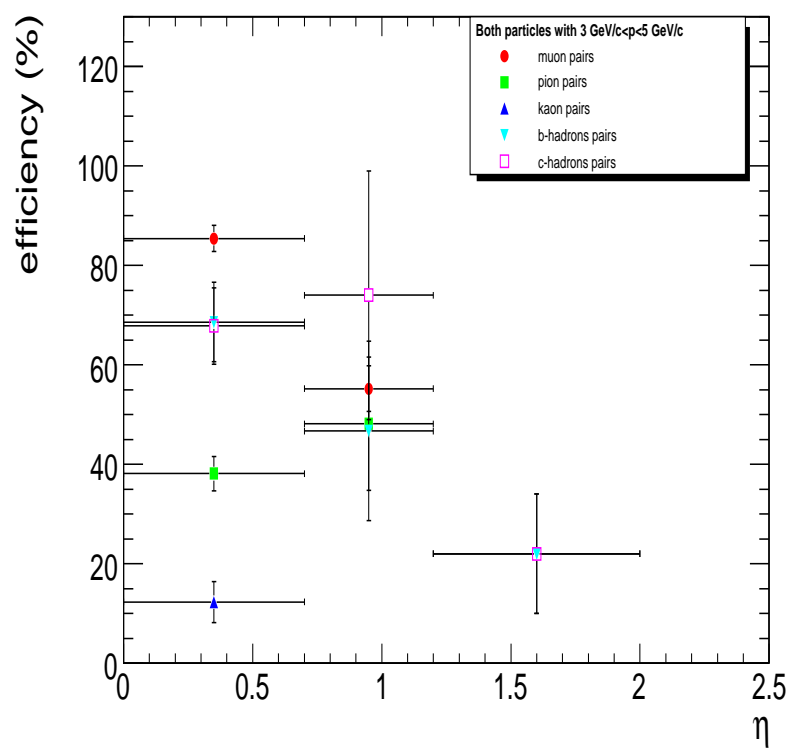

Figure 6: The dependence of the tracking efficiency on $|\eta|$ for pairs with both particles in $3 \mathrm{GeV}<p<5 \mathrm{GeV}$

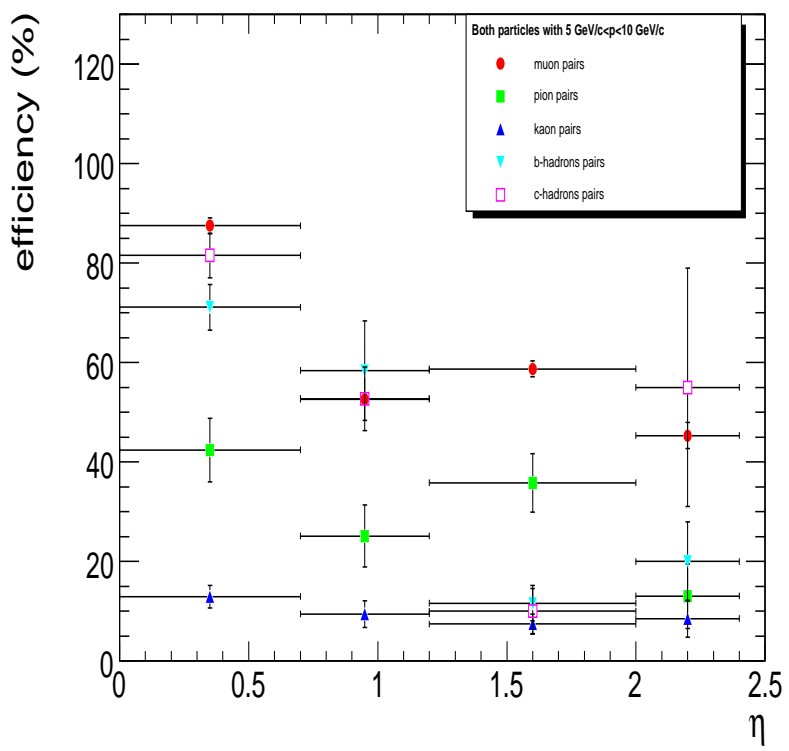

Figure 7: The dependence of the tracking efficiency on $|\eta|$ for pairs with both particles in $5 \mathrm{GeV}<p<10 \mathrm{GeV}$ 


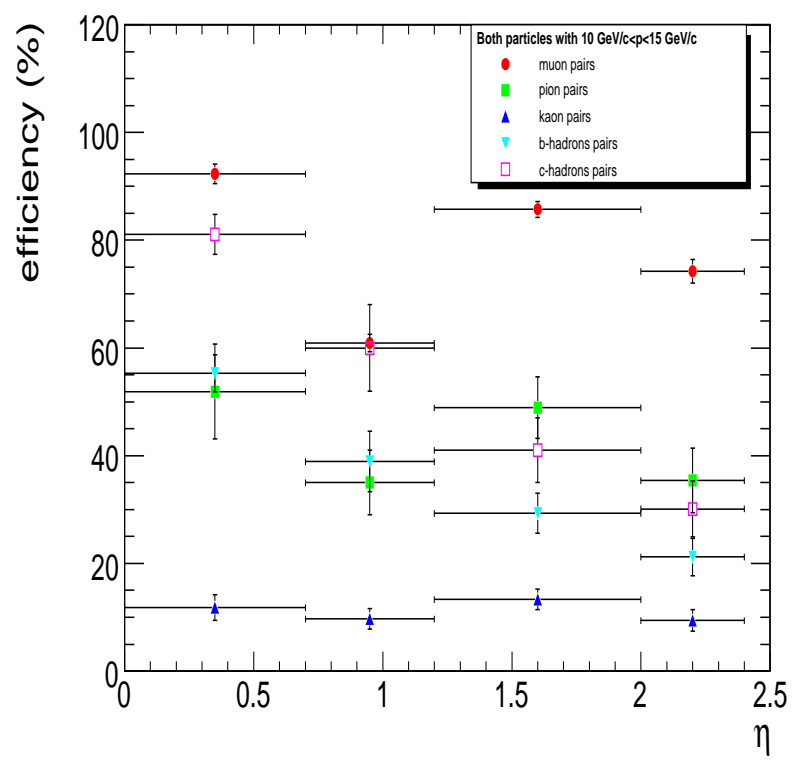

Figure 8: The dependence of the tracking efficiency on $|\eta|$ for pairs with both particles in $10 \mathrm{GeV}<p<15 \mathrm{GeV}$

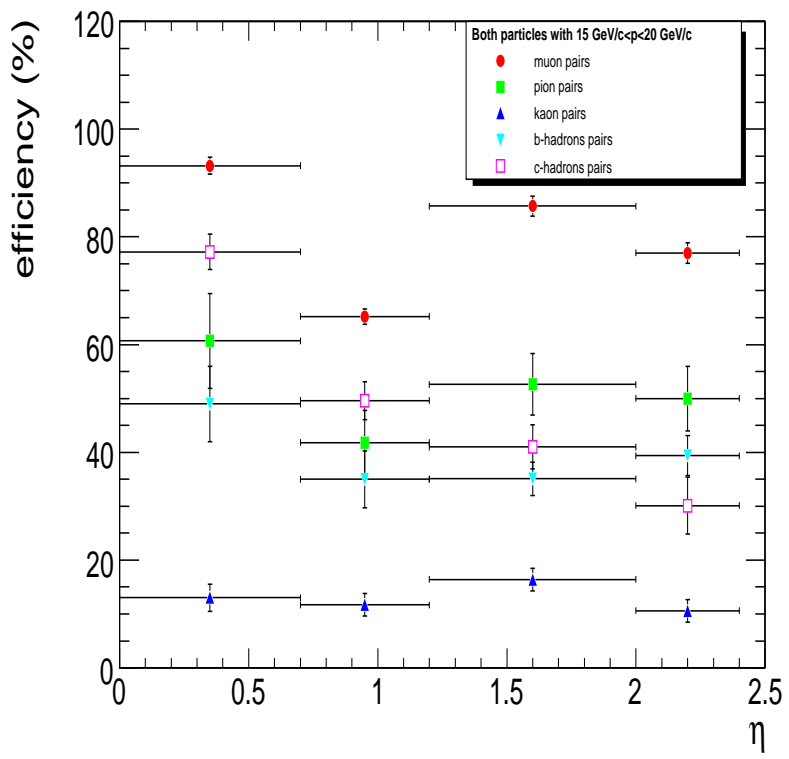

Figure 9: The dependence of the tracking efficiency on $|\eta|$ for pairs with both particles in $15 \mathrm{GeV}<p<20 \mathrm{GeV}$

The efficiency for pairs, shown in the Figs 6-9, is:

$$
\epsilon_{\text {pair }}=\epsilon_{\text {track }_{1}} \times \epsilon_{\text {track }_{2}} \times \epsilon_{\text {vertex }}
$$

To get the efficiency for the single particle, the square root of the efficiency for pairs of particles of the same type and in the same pseudorapidity and momentum bins is taken:

$$
\epsilon_{\text {single }}=\sqrt{\epsilon_{\text {pair }}}
$$

The main problem for pions and kaons is the statistics. The reconstruction in the tracker starts from the response of the level one and the level two triggers and takes the parameters of the level one/level two candidates as the initial parameters of the trajectory for propagation inside the tracker volume. The trigger selection efficiency for 
pions and kaons is very low. To accelerate the reconstruction processing, only particles that pass the sensitive muon volumes are saved in the database. This strategy allows to pass through level one and level two muon trigger only events that are able to give a response. The next optimization is in the list of allowed processes. The pions and kaons were forced to decay into muons and hadron electromagnetic processes, muon processes and electromagnetic processes are switched on. Open charm and bottom particles were generated using kinematical distributions (including correlations) of b- and c- quarks from [16] and PYTHIA [15].

The limited statistics of the reconstruction tables leads to additional systematical errors in the dimuon mass spectra. The expression for the weights and errors are in Sect. 5.4.

\subsubsection{Dependence of the reconstruction efficiency on multiplicity}

The dependence of the reconstruction efficiency on the multiplicity of events was studied with $\Upsilon$ resonance sample generated according [16] and $\mathrm{PbPb}$ events generated with HIJING [6]. The events are classified as barrel (both muons from $\Upsilon$ decay have $|\eta|<0.8$ and endcap (at least one muon in the pair has pseudorapidity $0.8<|\eta|<2.4$. The dependence of the reconstruction efficiency and of the purity of the reconstructed sample is shown in the Figs. 10,11 respectively.

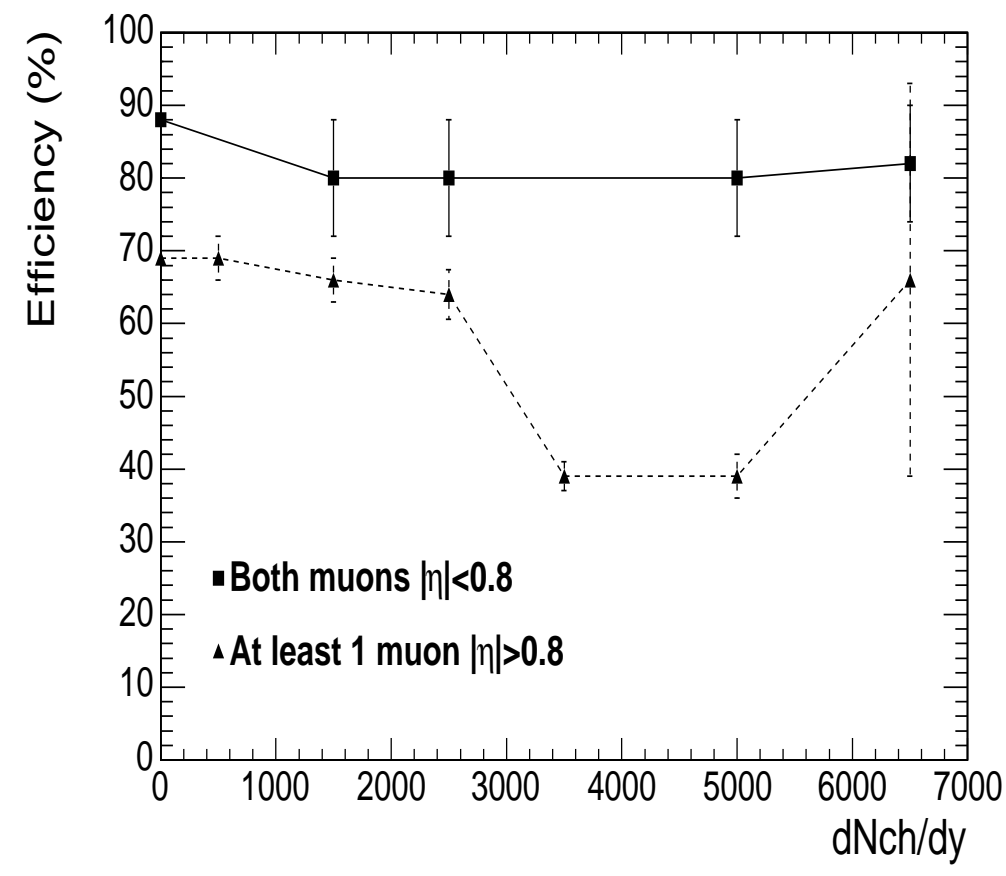

Figure 10: The dependence of the $\Upsilon$ reconstruction efficiency on the event multiplicity. Harder quality cuts, applied to the tracks with $|\eta|>0.8$ for the multiplicity $d N^{c h} / d y>2500$, results in the efficiency drop. 


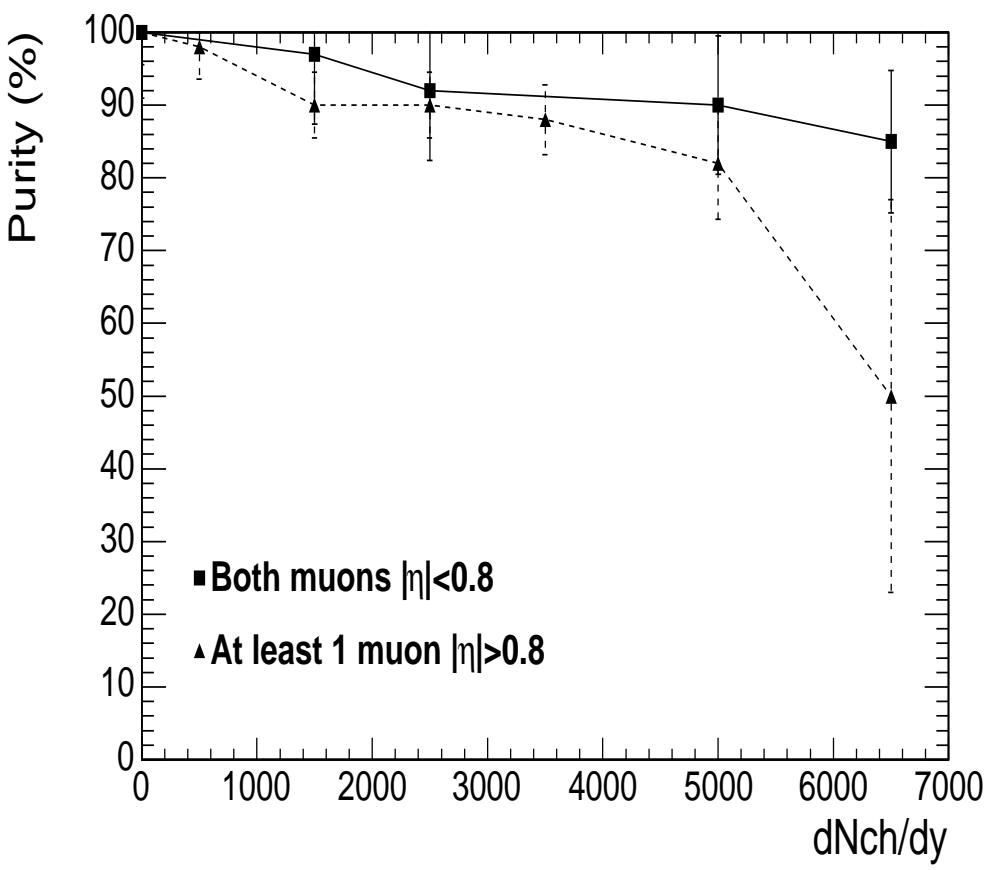

Figure 11: The dependence of the purity of the $\Upsilon$ reconstructed sample on the event multiplicity.

The efficiency in barrel does not depend heavily on the multiplicity of the event up to $d N^{c h} / d y=6500$ charged particles and the purity is kept above $80 \%$. For the events with at least one muon with $0.8<|\eta|<2.4$, stronger cuts were applied for the forward muons to keep the purity above $80 \%$ for multiplicities above $d N^{c h} / d y=2500$ charged particles. The counterpart is a drop in efficiency. Thus, the efficiency of $\Upsilon$ reconstruction is constant if both tracks have $|\eta|<0.8$. If at least one track in the pair has $|\eta|>0.8$, the efficiency is constant up to multiplicity $d N^{c h} / d y=2500$ and falls down to the other constant value for the higher multiplicities due to harder cuts on the track reconstruction quality. The other particle pair types $(\pi, K, b-, c-)$ follows the same dependence of the efficiency on the multiplicity [24], but with the different starting value at $d N^{c h} / d y=0$.

The tracker layers navigation [13] is simpler for endcap muons than for barrel muons. No gaps in layer configuration are allowed. This requirement can be changed easily for low multiplicity. For the high multiplicity (above $d N^{c h} / d y=2500$ ) the reconstruction algorithm in the endcap region may be more tricky. For the current study the requirement is to have a good purity for the reconstructed dimuon sample (Sect. 3.3.2).

One can apply another requirement to have a good efficiency rather than a good purity. Following this requirement for each triggered dimuon, a few prolongations in tracker will be assigned to one dimuon trigger candidate and only two tracks will correspond to the muon pair. The price will be an increase of the combinatorial background in the dimuon mass spectrum. In spite of the fact that the false muon pairs in the event will have masses from 0 to $20 \mathrm{GeV} / c^{2}$, part of them will appear under the resonance mass peak and affect the signal to background ratio.

\subsubsection{Mass resolution}

The mass resolution of the reconstructed muon pairs from $\Upsilon$ and $J / \Psi$ decay in the full pseudorapidity region $\left(|\eta|<2.4\right.$ ), without superimposing background events (Fig.12), is $86 \mathrm{MeV} / \mathrm{c}^{2}$ and $35 \mathrm{MeV} / \mathrm{c}^{2}$ correspondingly. For the barrel pseudorapidity region, where both muons are in $|\eta|<0.8$ range, the mass resolution is $54 \mathrm{MeV} / \mathrm{c}^{2}$.

The mass resolution depends slightly on the event multiplicity. Increasing the multiplicity from 0 to 2500 charged particles per rapidity unit increases the resolution of the reconstructed $\Upsilon$ mass from $86 \mathrm{MeV} / \mathrm{c}^{2}$ to $90 \mathrm{MeV} / \mathrm{c}^{2}$. With further increase of multiplicity to 5000 charged particles per rapidity unit the resolution decreases to 58 $\mathrm{MeV} / \mathrm{c}^{2}$ (Fig. 15). The reduction in the resolution is due to different treatment of endcap muons starting from the multiplicity $d N^{c h} / d y=2500$. For multiplicity larger than $d N^{c h} / d y=2500$ charged particles, a stronger cut is applied to the muons that intersect the tracker disks to keep the purity of the reconstructed dimuon sample. The efficiency of the forward muons is reduced but the purity is kept above $80 \%$ (Figs.10,11). The multiplicity will be estimated with pixel detectors and with full reconstruction in the tracker [22]. 
After applying the same cuts for all multiplicities, the mass resolution drops to $66 \mathrm{MeV} \pm 6 \mathrm{MeV}, 63 \mathrm{MeV} \pm$ $9 \mathrm{MeV}, 58 \mathrm{MeV} \pm 6 \mathrm{MeV}$ for $d N^{ \pm} / d y$ equal 0, 2500 and 5000, respectively (Figs.13-15). The gaussian fit is performed in the inteval $\pm 2 \times \sigma$ around the peak position. The residual dependence of the mass resolution on the event multiplicity reflects the ratio between events with both muons in the barrel part of the tracker and events with at least one muon intersecting the tracker disks. This ratio amounts $0.25,0.28,0.34$ for $d N^{ \pm} / d y$ equal 0,2500 and 5000 , respectively. Tracks in the endcap have up to two times worse momentum resolution than barrel tracks [22].

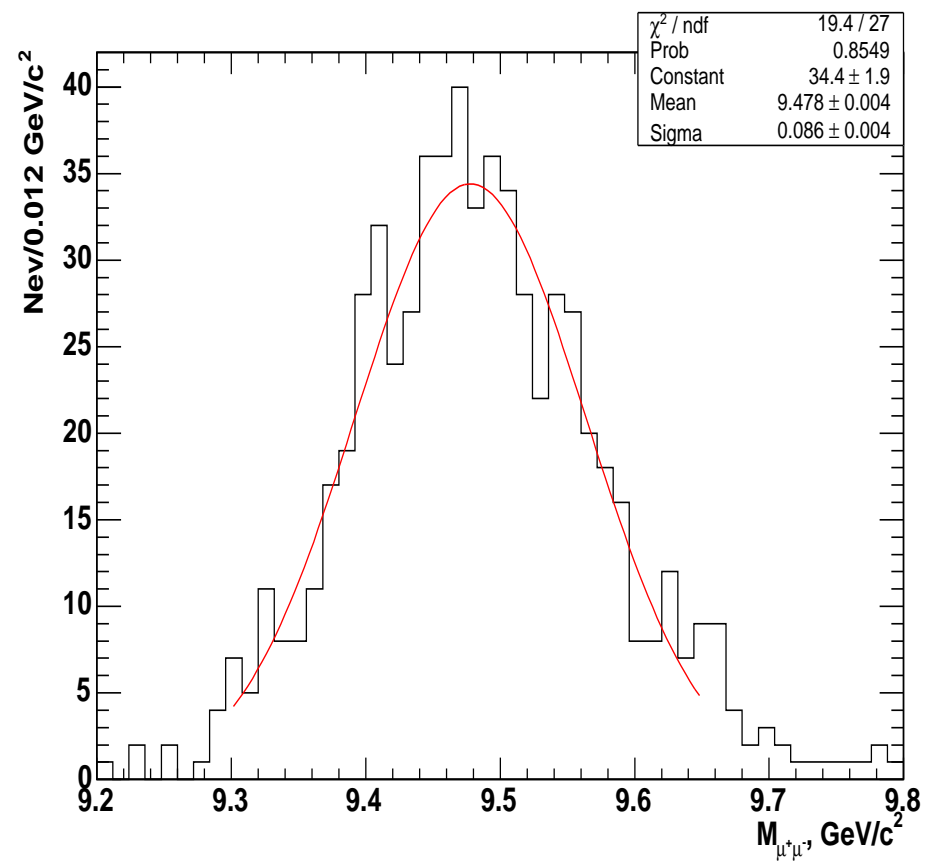

Figure 12: The reconstructed $\Upsilon$ mass in $\mathrm{PbPb}$ event with multiplicity $d N^{ \pm} /\left.d y\right|_{y=0}=0$. Both muons are in $|\eta|<2.4$. 


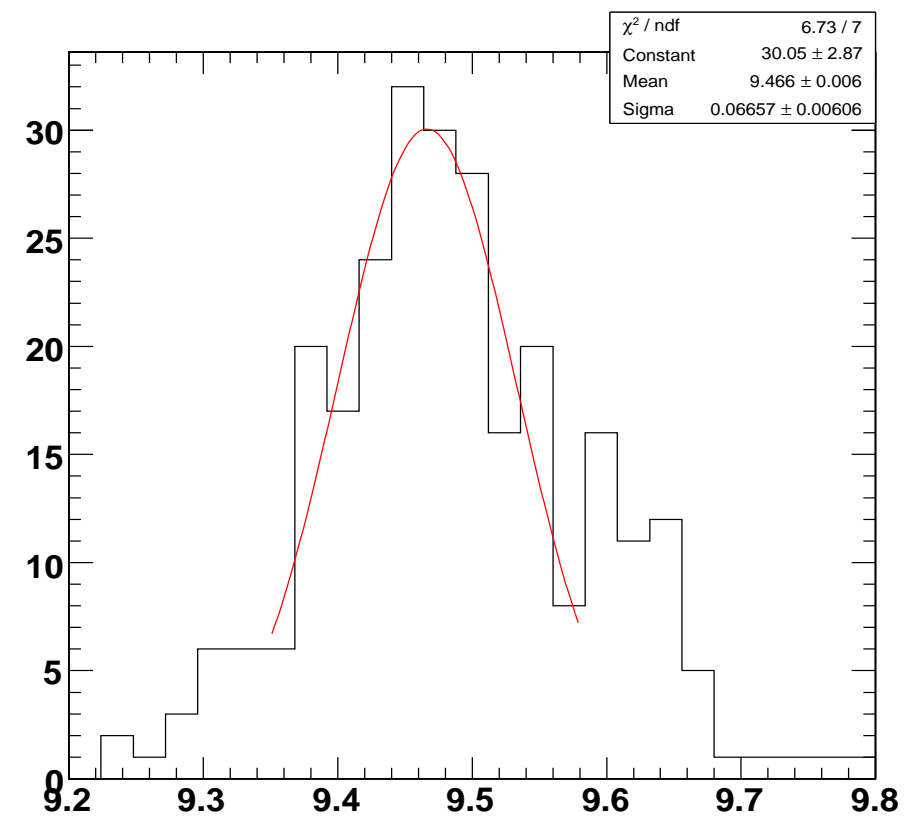

Figure 13: The reconstructed $\Upsilon$ mass in $\mathrm{PbPb}$ event with multiplicity $d N^{ \pm} /\left.d y\right|_{y=0}=0$. Both muons are in $|\eta|<2.4$.

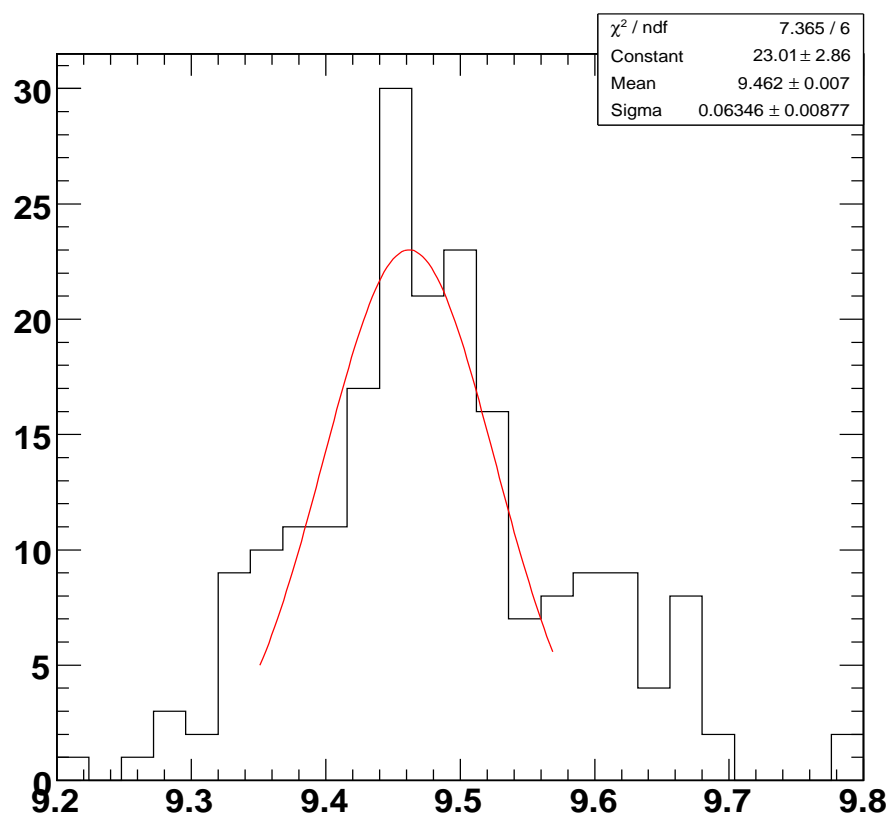

Figure 14: The reconstructed $\Upsilon$ mass in PbPb event with multiplicity $d N^{ \pm} /\left.d y\right|_{y=0}=2500$. Both muons are in $|\eta|<2.4$. 


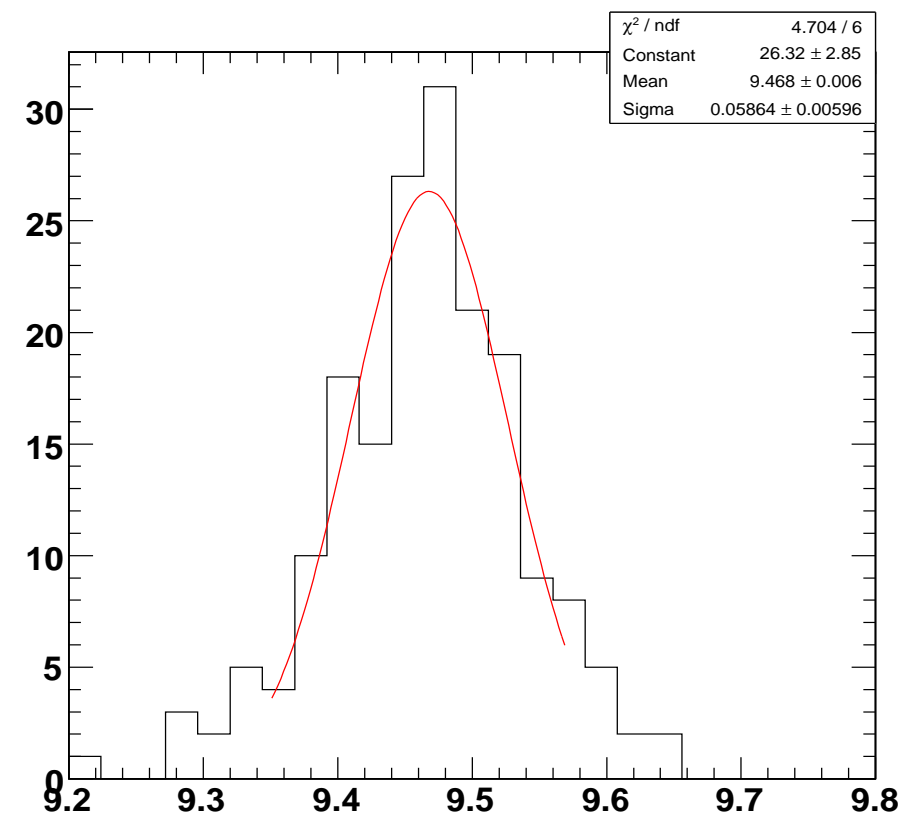

Figure 15: The reconstructed $\Upsilon$ mass in $\mathrm{PbPb}$ event with multiplicity $d N^{ \pm} /\left.d y\right|_{y=0}=5000$. Both muons are in $|\eta|<2.4$.

Two different approaches to introduce mass resolution into fast Monte-Carlo are studied.

In the first method the value $85 \mathrm{MeV} / \mathrm{c}^{2}$ is chosen in the fast Monte-Carlo program for the $\Upsilon$ mass range and 37 $\mathrm{MeV} / \mathrm{c}^{2}$ is used for the $\mathrm{J} / \psi$ mass range for the full pseudorapidity and multiplicity ranges. One has to mention that the dependence of the mass resolution on multiplicity was studied only for the upsilon mass range. In the second method, the mass resolution is taken as $\sigma=0.009 \times M$, where $\mathrm{M}$ is the mass of the pair of particles of any type. Both methods are verified with detailed simulation (Figs.41,42). No significant differences are observed and the first method is used to provide mass plots.

\subsubsection{5 $p_{T}$ and $\eta$ spectra after reconstruction in the tracker}

The $p_{T}$ and $\eta$ spectra of the $\Upsilon$ resonance, reconstructed in the tracker, are shown as hatched histograms in Figs. 1621 for the different multiplicities of $\mathrm{Pb}-\mathrm{Pb}$ events.

The empty histograms show the $p_{T}$ and $\eta$ spectra of the $\Upsilon$ resonance, reconstructed in the muon stations only, with an OR of the level 1 and the level 2 trigger condition with the additional request of two opposite sign tracks (Sect.3.3.2).

The difference between empty and hatched histograms shows the efficiency to reconstruct the $\Upsilon$ resonance depending on $p_{T}$ and $\eta$. 


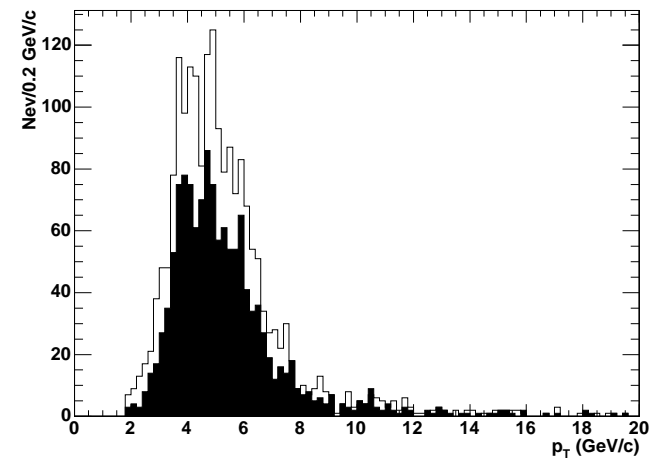

Figure 16: The reconstructed $p_{T}$ of the $\Upsilon$ resonance without $\mathrm{PbPb}$ event. Both muons are in $|\eta|<2.4$.

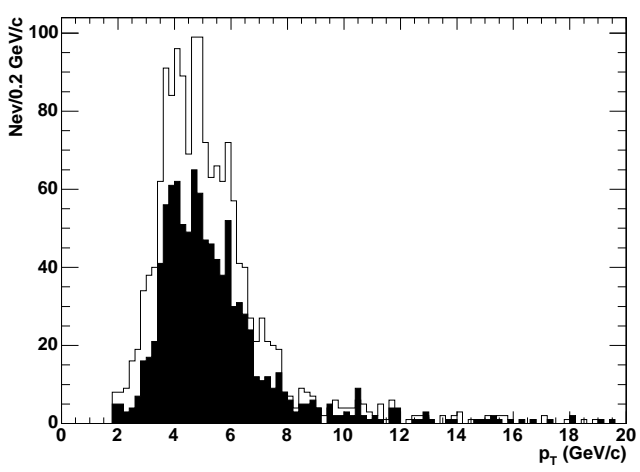

Figure 18: The reconstructed $p_{T}$ of the $\Upsilon$ resonance in $\mathrm{PbPb}$ event with multiplicity $d N^{ \pm} /\left.d y\right|_{y=0}=$ 1500. Both muons are in $|\eta|<2$.4.

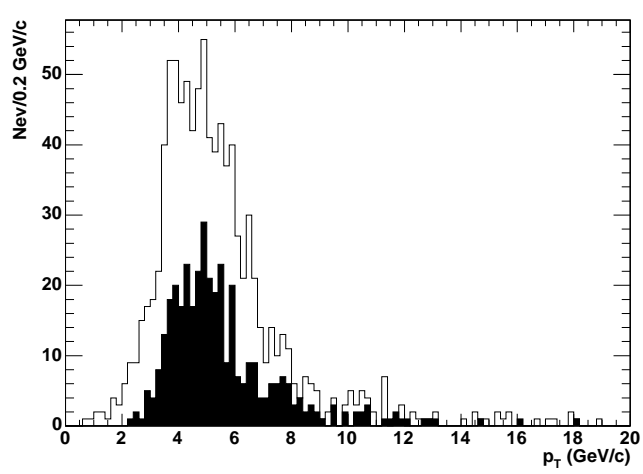

Figure 20: The reconstructed $p_{T}$ of the $\Upsilon$ resonance in $\mathrm{PbPb}$ event with multiplicity $d N^{ \pm} /\left.d y\right|_{y=0}=$ 5000. Both muons are in $|\eta|<2.4$.

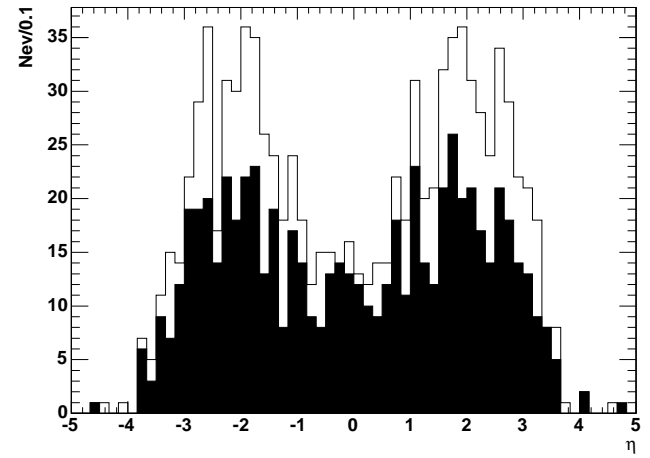

Figure 17: The reconstructed $\eta$ of the $\Upsilon$ resonance without $\mathrm{PbPb}$ event. Both muons are in $|\eta|<2.4$.

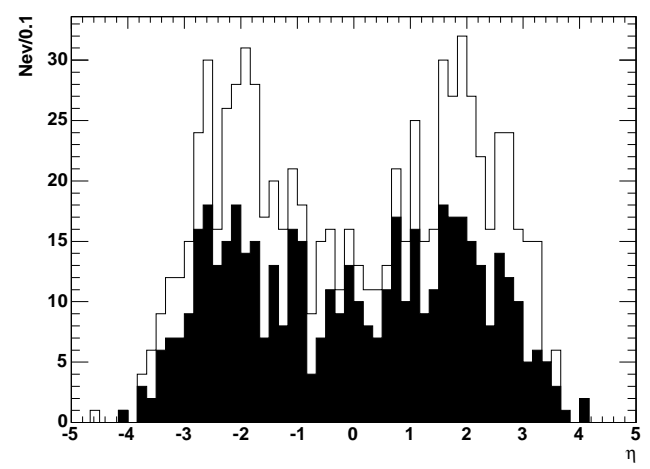

Figure 19: The reconstructed $\eta$ of the $\Upsilon$ resonance in $\mathrm{PbPb}$ event with multiplicity $d N^{ \pm} /\left.d y\right|_{y=0}=$ 1500. Both muons are in $|\eta|<2.4$.

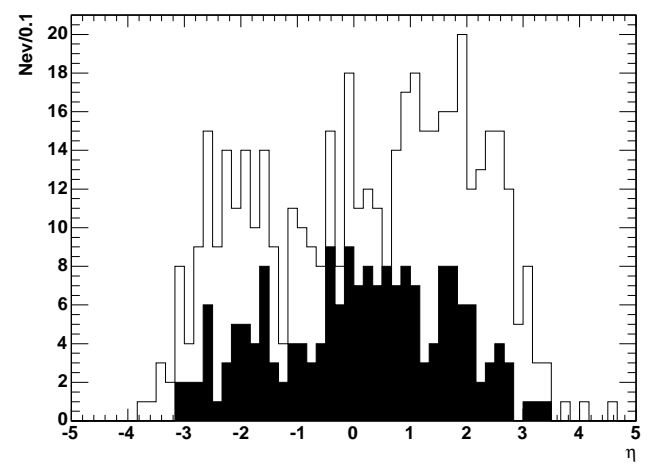

Figure 21: The reconstructed $\eta$ of the $\Upsilon$ resonance in $\mathrm{PbPb}$ event with multiplicity $d N^{ \pm} /\left.d y\right|_{y=0}=$ 5000. Both muons are in $|\eta|<2.4$. 


\section{Fast Monte Carlo}

\subsection{Event simulation with fast Monte Carlo}

In each $\mathrm{Pb}+\mathrm{Pb}$ collision characterized by its impact parameter, all 5 resonances $J / \psi \psi^{\prime}$ and the 3 states of the $\Upsilon$ family, generated according to [16], are superimposed to the background made of $\pi$ 's, $K$ 's and muons coming from open heavy quark pair production. The resonances are forced to decay into muons.

All muons which are within the acceptance of the CMS detector, i.e $\mathrm{P}>2.8 \mathrm{GeV} / c$ and $|\eta|<2.4$ are recorded with the following quantities: momentum, pseudorapidity, charge and origin. The muons are weighted according to:

$$
W_{\mu}=W_{\text {origin }} * W_{\text {rec }}
$$

where $W_{\text {origin }}$ is equal to 1 for the background muons and to $\sigma$ (quarkonium) $/ \sigma(\mathrm{PbPb})$ for the muons from quarkonia decay with $\sigma(\mathrm{PbPb})=8$ barns. $W_{\text {rec }}$ is the track reconstruction efficiency depending on the multiplicity, momentum, pseudorapidity and purity. Each muon candidate is also characterized by the trigger efficiencies $W_{t r i g}^{L V L 1}$ and $W_{\text {trig }}^{L V L}$.

The invariant dimuon mass is calculated and smeared according to a Gaussian law with $\sigma=85 \mathrm{MeV} / \mathrm{c}^{2}$ for masses above $5 \mathrm{GeV} / \mathrm{c}^{2}$ and $\sigma=37 \mathrm{MeV}$ for masses below $5 \mathrm{GeV} / \mathrm{c}^{2}$.

The weight of the pair is given by:

$$
W\left(\mu_{1} \mu_{2}\right)=W_{\mu 1} * W_{\mu 2} * W_{\text {trigger }}
$$

where $W_{\text {trigger }}$ is the combination of the trigger efficiencies of Level 1 and Level 2 of both muons. The detailed expression for the weights is in Sect. 5.4. Since the muon charge is registered it is possible to separate like sign muon pairs from the opposite sign pairs.

This method allows to study $\approx 5010^{6} \mathrm{~Pb}+\mathrm{Pb}$ collisions at any impact parameter.

\subsection{Quarkonia acceptance}

After applying the L1 and L2 trigger tables (Sect. 3.3.1) to the $\Upsilon$ and $J / \psi$ muon decay, generated according to [16], the integrated acceptance is about $1.3 \%$ for the $J / \psi$ and $26 \%$ for the $\Upsilon$. These figures must be compared to the acceptances obtained with a detailed trigger simulation (Sect.3.3.2) which gives $1 \% \pm 0.06 \%$ for the $J / \psi$ resonance and $21 \% \pm 2 \%$ for the $\Upsilon$.

Misidentification of muon charge by the low quality L1 muon trigger is not taking into account. For low momentum muon candidate with low quality assumption the misidentification of muon charge depends on $\eta$ and may reach $10-15 \%$ in the region $\eta>2$. Level two muon trigger partially compensates the muon charge misidentification. After applying L3 dimuon trigger efficiency tables, the systematical shift between fast Monte-Carlo simulation and detailed simulation is less then $10 \%$ (Sect. 5.5.2) and does not exceed systematical errors (Sect. 5.4).

With the transverse momentum $\left(\mathrm{P}_{T}\right)$ distributions at the generation level for $J / \psi$ and $\Upsilon$ shown in the left half of fig. 22, the $\mathrm{P}_{T}$ dependence of the acceptance is shown in the right half of the same figure. The curves are integrated over the pseudorapidity range $(|\eta|<2.4)$ including barrel and endcap regions. They indicate a different trend between the resonances. Because of its relatively low rest mass the $J / \psi$ can be detected only when its $\mathrm{P}_{T}$ is above $4 \mathrm{GeV} / c$. This threshold is even higher, at $6-7 \mathrm{GeV} / c$, if the detection is restricted to the barrel part of the detector. The situation looks different for the $\Upsilon$ for which the acceptance is as large as $40 \%$ for $\mathrm{P}_{T} \rightarrow 0$ and stays almost flat at 15\% (full CMS) or 5\% (barrel) for $\mathrm{P}_{T}$ above $4 \mathrm{GeV} / c$. 

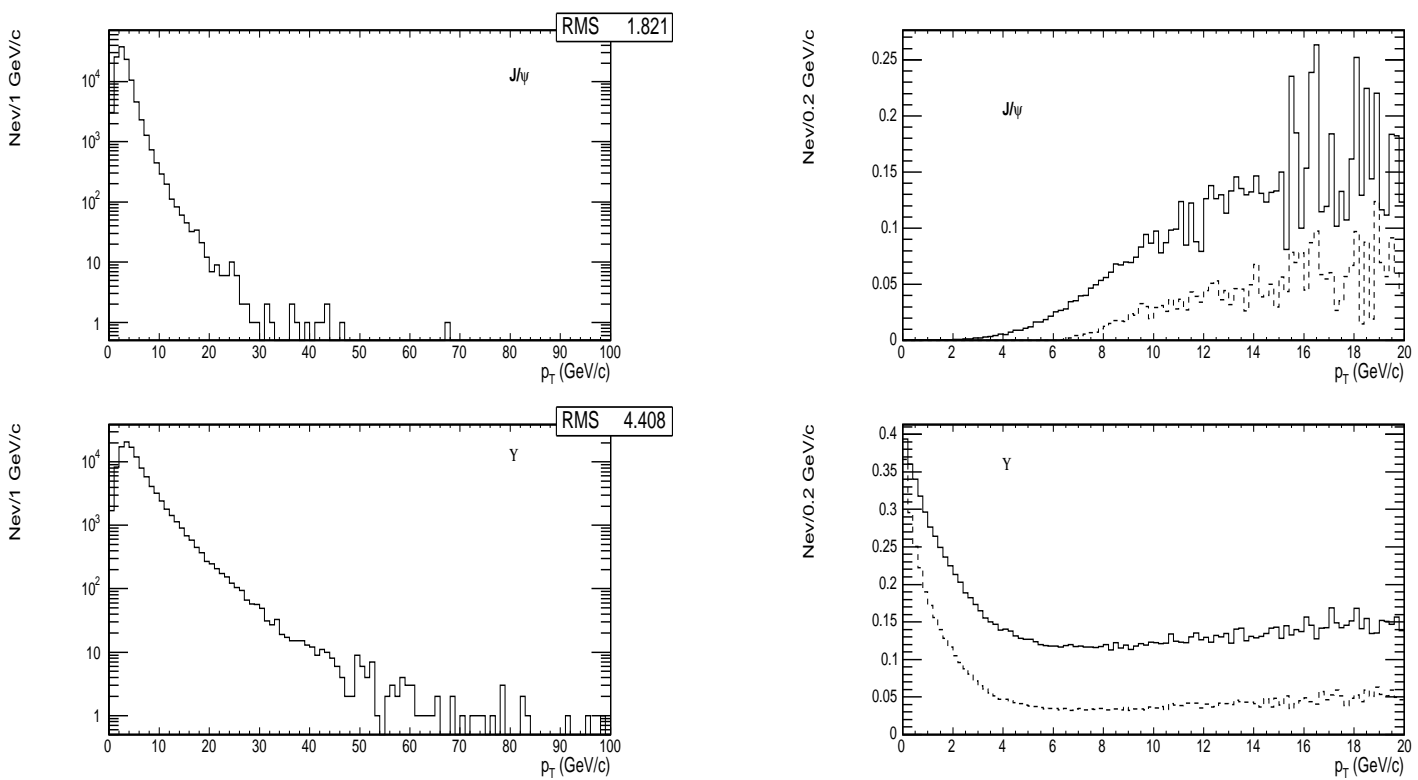

Figure 22: On the left part, the $\mathrm{P}_{T}$ distributions of $J / \psi$ (up) and $\Upsilon$ (down)at the generation level. On the right part, the $\mathrm{P}_{T}$ dependence of accepted quarkonia in full CMS detector (full line) and in the barrel alone (dashed line).

\section{Simulation of quarkonia data}

\subsection{Dimuon invariant mass}

About $50 \times 10^{6} \mathrm{~Pb}+\mathrm{Pb}$ collisions were simulated depending on the impact parameter in each multiplicity set. Recorded muons are combined to form pairs and their invariant mass is calculated. The mass is smeared as described above. Opposite sign, as well as like sign muon pair masses, properly weighted (Sect. 4.1), are plotted. Each contribution to the mass distribution can be histogrammed separately. The signal distribution due to quarkonia can be distinguished from dimuons of a precise background origin $\pi / K$-decay muons or $Q \bar{Q}$ pairs or mixed origin, i.e. one muon from $\pi / K$-decay the other from $Q \bar{Q}$ pairs.

The mass spectrum obtained is scaled to $0.5 \mathrm{nb}^{-1}$ which corresponds to the integrated luminosity collected after one month of $\mathrm{Pb}$ beams with an average luminosity $\mathcal{L}=4 \times 10^{26} \mathrm{~cm}^{-2} \mathrm{~s}^{-1}$ and 0.5 machine efficiency.

Two mass regions are considered:

the $J / \psi$ mass region with $2.0<M_{\mu \mu}<4.5 \mathrm{GeV} / c^{2}$

the $\Upsilon$ mass region with $8.5<M_{\mu \mu}<11.0 \mathrm{GeV} / c^{2}$

Figure 23 shows the invariant mass distributions corresponding to $0.5 \mathrm{nb}^{-1}$ and multiplicity $\mathrm{d} N^{ \pm} / \mathrm{d} \eta=5000$ in the $J / \psi$ mass region and $\Upsilon$ mass region respectively. The width of a histogram bin is $0.025 \mathrm{GeV} / c^{2}$. The distributions have been obtained for the high multiplicity set. The different background contributions are also shown, 'h' holds for $\pi / K$-decay. In both mass regions the dominant background is due to the $\pi / K$-decay, either as $\pi / K$-decay muon pairs or mixed origin. Clearly more than $90 \%$ of the background is made of uncorrelated muon pairs.

A prominent $J / \psi$ peak is observed contrary to the $\psi^{\prime}$, for which the low production cross section prevents to emerge from the background. In the $\Upsilon$ mass region the two states $\Upsilon$ and $\Upsilon^{\prime}$ are well separated thanks to the good mass resolution. The third state, $\Upsilon^{\prime \prime}$, will be less easily observed if the background level is so high. 

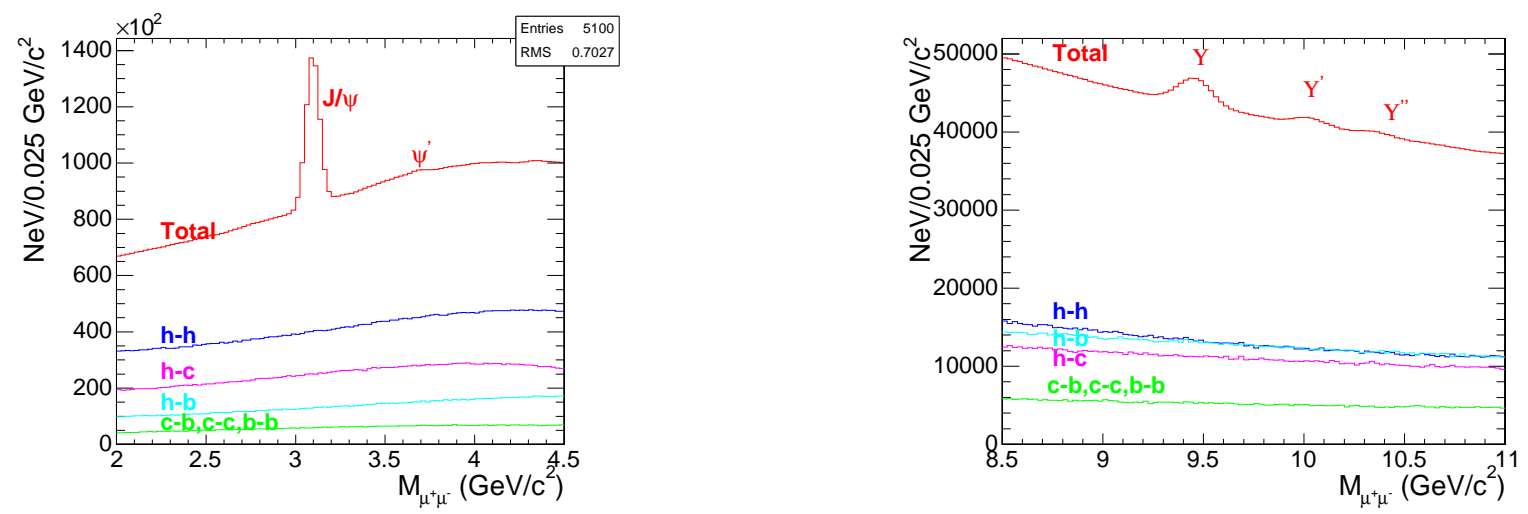

Figure 23: Invariant mass of opposite sign muon pairs with $\mathrm{d} N^{ \pm} / \mathrm{d} \eta=5000$, in the $J / \psi$ region (left) and $\Upsilon$ mass region (right). The main contributions of the background are shown. The sign $\mathrm{h}$ holds for $\pi, \mathrm{K}$ decay. Both muons have $|\eta|<2.4$.

With the assumption of smaller multiplicity, $\mathrm{d} N^{ \pm} / \mathrm{d} \eta=2500$, see figure 24 , the situation is more favourable. A bump corresponding at the $\psi^{\prime}$ mass is observed. In the $\Upsilon$ mass region the three resonant states are well seen and well separated. The main difference with the high multiplicity set is due to the background. At low multiplicity the mixed origin background is dominant and twice as high as the $\pi / K$-decay component.
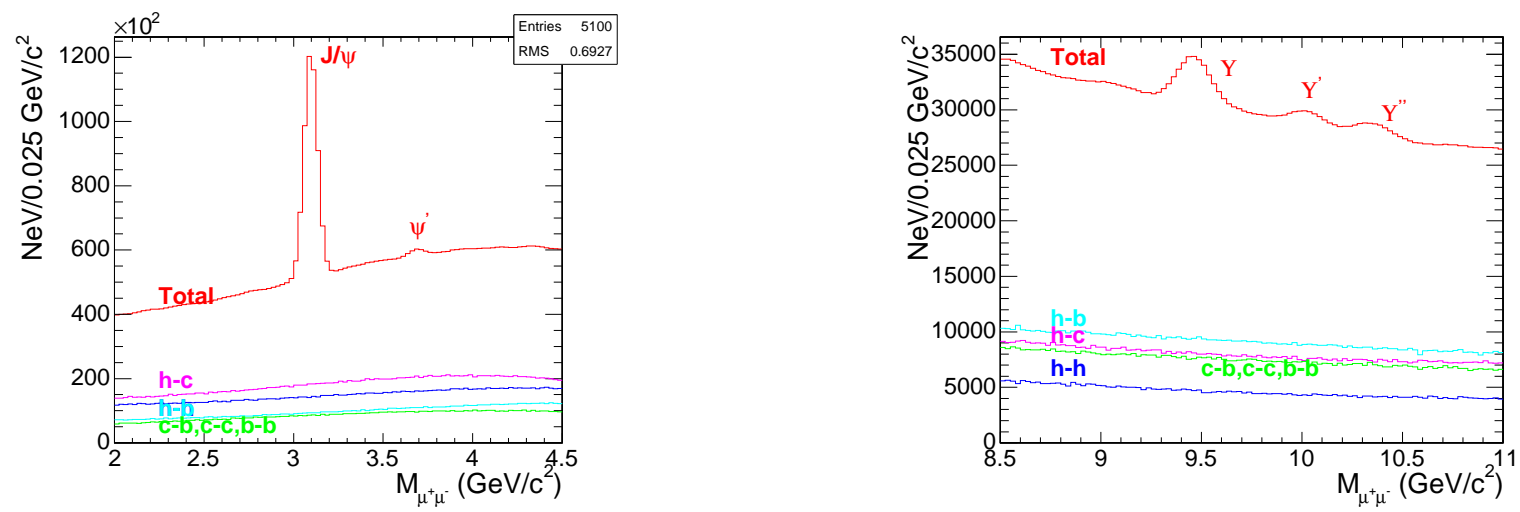

Figure 24: Invariant mass of opposite sign muon pairs with $\mathrm{d} N^{ \pm} / \mathrm{d} \eta=2500$, in the $J / \psi$ region (left) and $\Upsilon$ mass region (right) The main contributions of the background are shown. The sign h holds for $\pi, \mathrm{K}$ decay. Both muons have $|\eta|<2.4$.

The background due to uncorrelated muon pairs must also be present in the mass distribution of like sign muon pairs. Assuming that the correlated like sign muon pairs are in a very low amount, the uncorrelated background can be subtracted from the opposite sign dimuon mass distribution leaving the signal, i.e the dimuons from quarkonia decay. In each bin of mass the signal is given by [17]:

$$
N^{\mathrm{Sig}}=N^{+-}-2 \sqrt{N^{++} N^{--}}
$$

where $\mathrm{N}^{+-}, \mathrm{N}^{++}$and $\mathrm{N}^{--}$are the combinations of opposite sign pairs and positive and negative like sign pairs respectively in a given mass interval.

The track reconstruction in barrel $(|\eta|<0.8)$ gives a better track momentum resolution and as a consequence the best dimuon mass resolution. Figures 25, 26 show the mass distributions for the dimuons with both particles in $|\eta|<0.8$. Like sign dimuon mass spectra $\left(2 \sqrt{N^{++} N^{--}}\right)$are presented in the same plots. Limitation of the $\eta$ range leads to the better separation of the $\Upsilon$ states but with lower statistics.

Figures 27-30 presents the mass distributions resulting from this subtraction. The $\Upsilon$ 's clearly emerge above a very low background. Figures 31-34 presents the mass distributions resulting from the subtraction. Both particles are in $|\eta|<0.8$ and a mass resolution of $54 \mathrm{MeV} / \mathrm{c}^{2}$. 

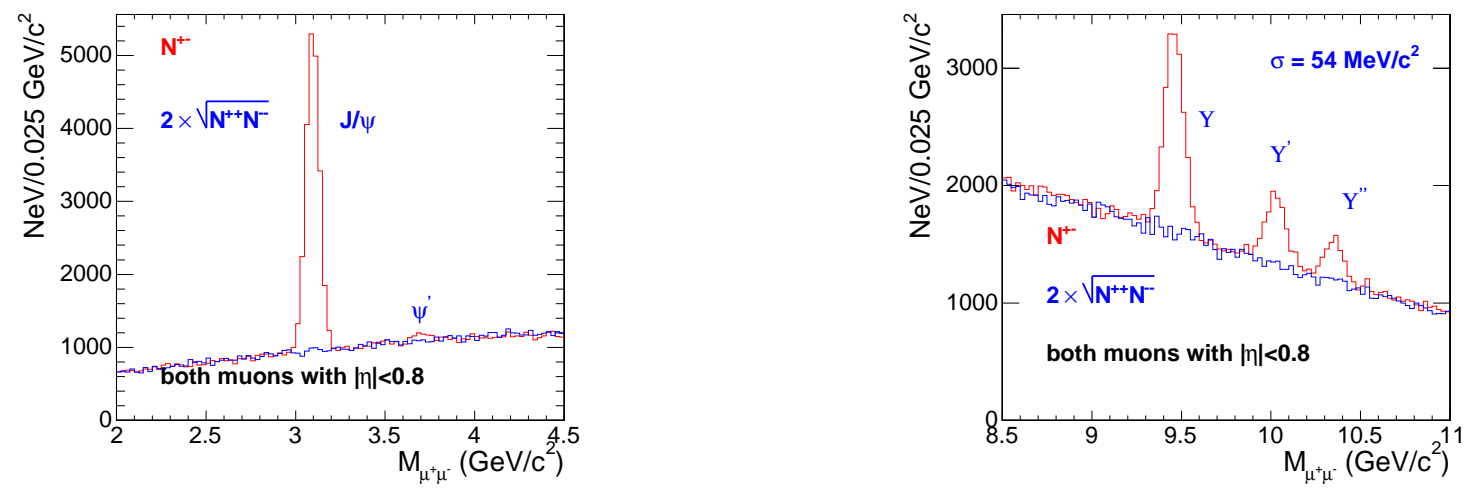

Figure 25: Invariant mass of opposite sign muon pairs with $\mathrm{d} N^{ \pm} / \mathrm{d} \eta=2500$, in the $J / \psi$ region (left) and $\Upsilon$ mass region (right). Both muons have $|\eta|<0.8$.
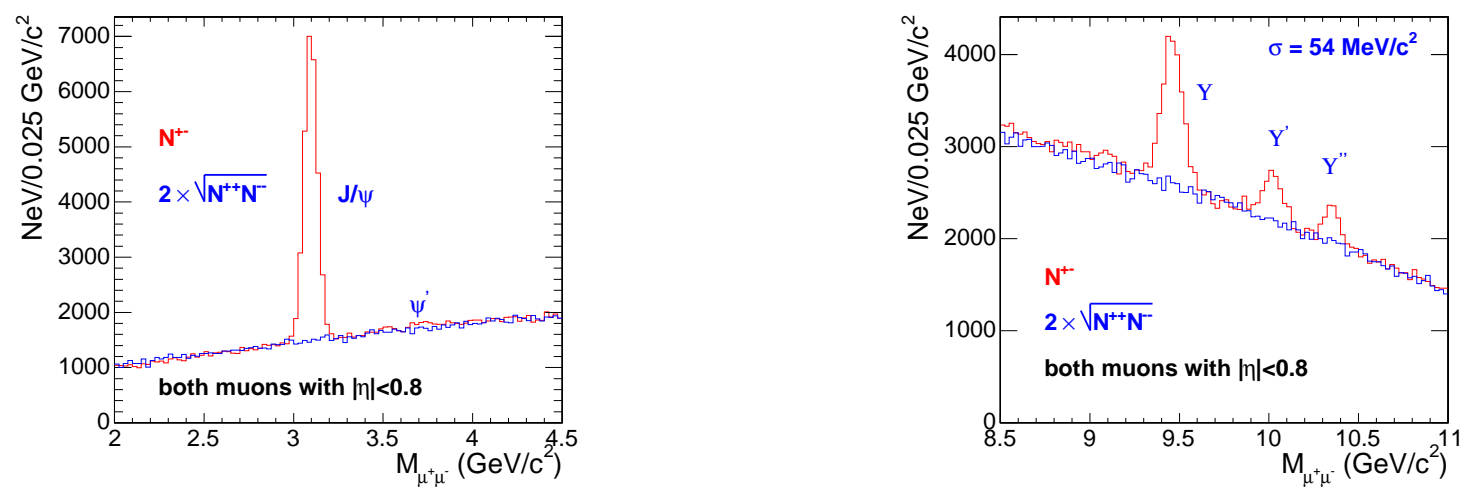

Figure 26: Invariant mass of opposite sign muon pairs with $\mathrm{d} N^{ \pm} / \mathrm{d} \eta=5000$, in the $J / \psi$ region (left) and $\Upsilon$ mass region (right). Both muons have $|\eta|<0.8$

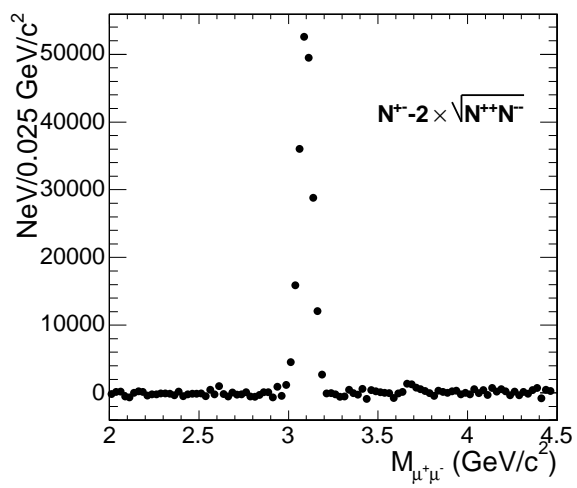

Figure 27: Signal after background subtraction in the $J / \psi$ region with $\mathrm{d} N^{ \pm} / \mathrm{d} \eta=5000$. Both muons have $|\eta|<2.4$ 


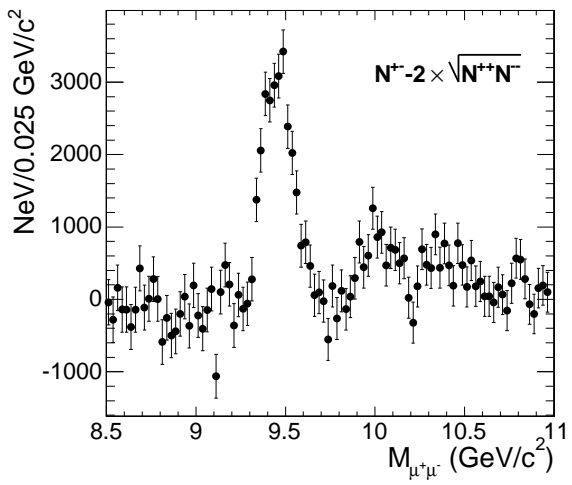

Figure 28: Signal after background subtraction in the $\Upsilon$ region with $\mathrm{d} N^{ \pm} / \mathrm{d} \eta=5000$. Both muons have $|\eta|<2.4$

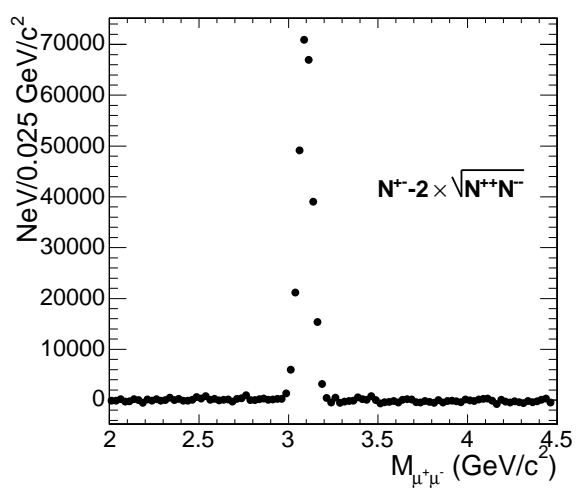

Figure 29: Signal after background subtraction in the $J / \psi$ region with $\mathrm{d} N^{ \pm} / \mathrm{d} \eta=2500$. Both muons have $|\eta|<2.4$

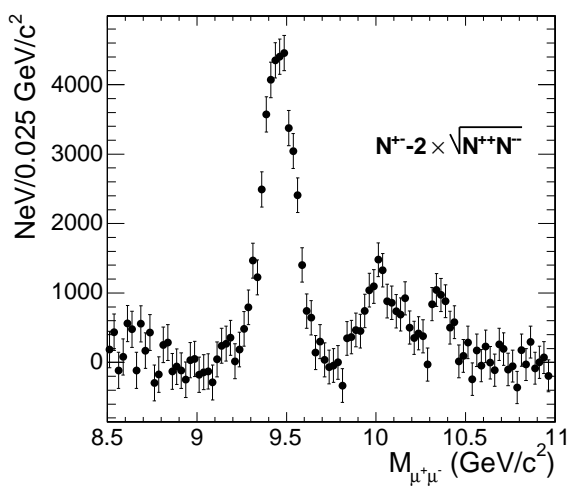

Figure 30: Signal after background subtraction in the $\Upsilon$ region with $\mathrm{d} N^{ \pm} / \mathrm{d} \eta=2500$. Both muons have $|\eta|<2.4$ 


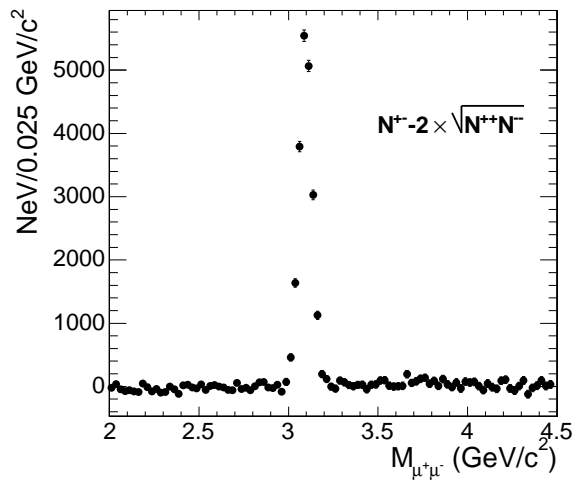

Figure 31: Signal after background subtraction in the $J / \psi$ region with $\mathrm{d} N^{ \pm} / \mathrm{d} \eta=5000$. Both muons have $|\eta|<0.8$.

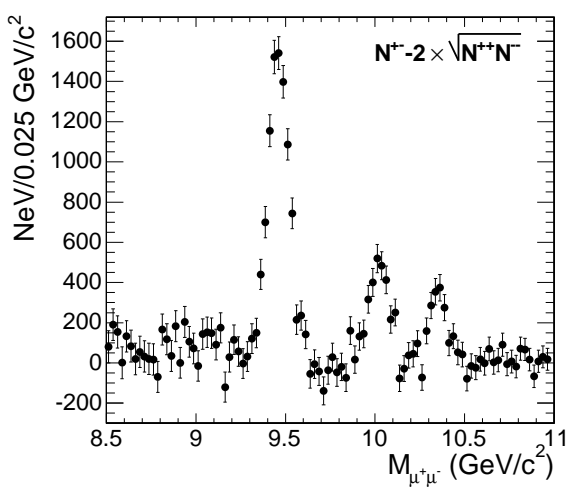

Figure 32: Signal after background subtraction in the $\Upsilon$ region with $\mathrm{d} N^{ \pm} / \mathrm{d} \eta=5000$. Both muons have $|\eta|<0.8$.

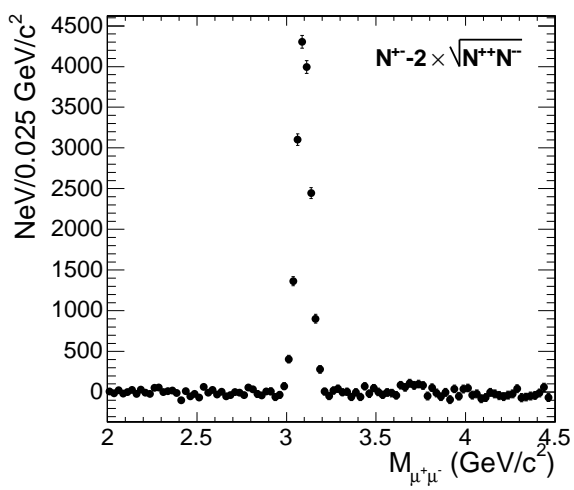

Figure 33: Signal after background subtraction in the $J / \psi$ region with $\mathrm{d} N^{ \pm} / \mathrm{d} \eta=2500$. Both muons have $|\eta|<0.8$. 


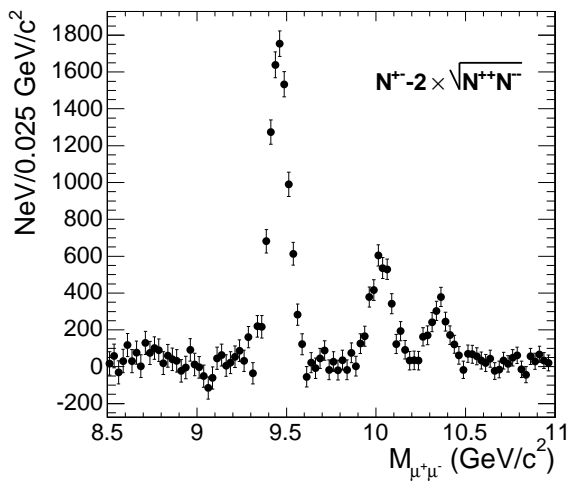

Figure 34: Signal after background subtraction in the $\Upsilon$ region with $\mathrm{d} N^{ \pm} / \mathrm{d} \eta=2500$. Both muons have $|\eta|<0.8$.

\subsection{Signal/Background ratio and statistics}

The statistics of $J / \psi$ and $\Upsilon$ 's expected in one month of data taking are shown in the tables 3 . The Signal/Background ratios are also indicated. These quantities have been calculated for an integrated luminosity of $0.5 \mathrm{nb}^{-1}$ assuming an average luminosity $\mathcal{L}=4 \times 10^{26} \mathrm{~cm}^{-2} \mathrm{~s}^{-1}$ and a machine efficiency of 0.5 . In the tables, the background and reconstructed resonance numbers are in a mass interval $\pm \sigma$ where $\sigma$ is the mass resolution.

Since the reconstruction efficiencies depend on the multiplicity of tracks it is not surprising to have an increased number of reconstructed resonances at lower $\mathrm{d} N^{ \pm} / \mathrm{d} \eta$. The expected statistics are large enough to allow further offline analysis for example in correlation with the centrality of the collision or the transverse momentum of the resonance.

Table 3: The signal-to-background ratio, $\mathrm{S} / \mathrm{B}$, and the number of $J / \psi$ expected in $0.5 \mathrm{nb}^{-1}$ with the 2 sets of multiplicity

\begin{tabular}{|l|r|r|r|r|r|r|}
\cline { 2 - 7 } \multicolumn{1}{c|}{} & $\mathrm{S} / \mathrm{B}(J / \psi)$ & $\mathrm{N}(J / \psi)$ & $\mathrm{S} / \mathrm{B}(\Upsilon)$ & $\mathrm{N}(\Upsilon)$ & $\mathrm{N}\left(\Upsilon^{\prime}\right)$ & $\mathrm{N}\left(\Upsilon^{\prime \prime}\right)$ \\
\hline $\mathrm{d} N^{ \pm} / \mathrm{d} \eta=5000$ & 0.6 & 140000 & 0.07 & 20000 & 5900 & 3500 \\
\hline $\mathrm{d} N^{ \pm} / \mathrm{d} \eta=2500$ & 1.2 & 180000 & 0.12 & 25000 & 7300 & 4400 \\
\hline
\end{tabular}

The signal-to-background ratio and the number of events collected in one month are shown in Tables 4 for the dimuons in $J / \psi$ and $\Upsilon$ mass regions with both particles in $|\eta|<0.8$ region.

Table 4: The signal-to-background ratio, $\mathrm{S} / \mathrm{B}$, and the number of $J / \psi$ expected in $0.5 \mathrm{nb}^{-1}$ with the 2 sets of multiplicity. Both particles with $|\eta|<0.8$.

\begin{tabular}{|l|r|r|r|r|}
\cline { 2 - 5 } \multicolumn{1}{c|}{} & $\mathrm{S} / \mathrm{B}(J / \psi)$ & $\mathrm{N}(J / \psi)$ & $\mathrm{S} / \mathrm{B}(\Upsilon)$ & $\mathrm{N}(\Upsilon)$ \\
\hline $\mathrm{d} N^{ \pm} / \mathrm{d} \eta=5000$ & 2.75 & 12636 & 0.52 & 6032 \\
\hline $\mathrm{d} N^{ \pm} / \mathrm{d} \eta=2500$ & 4.5 & 11648 & 0.97 & 6396 \\
\hline
\end{tabular}

The signal-to-background ratios is equal to 1.2 in the low multiplicity set, 0.6 in the high multiplicity set for $\mathrm{J} / \psi$ and $0.12,0.07$ respectively for $\Upsilon$.

One has to remark, that taking into account hot matter interactions and assuming the same magnitudes as in RHIC, the $\mathrm{J} / \psi$ could be suppressed by a factor of three (including cold matter effects, but excluding regeneration possibility), while the background (both from heavy and light quark production) should decrease by a factor of from two to five depending on $p_{T}$ for the accepted kinematical range $(p>3 \mathrm{GeV} / \mathrm{c},|\eta|<2.4)$ [25]. Thus, the signal-to-backround ratio at the $\mathrm{J} / \psi$ mass should increase anyway. 


\subsection{Muon kinematical selection}

The above S/B ratios are relative to the entire CMS detector. A further offline analysis might improve these ratios. The muon detection space can be divided into three regions:

- a) both muons are detected in the barrel, $\left|\eta_{\mu}\right|<1.2$

- b) both muons are detected in the endcaps, $\left|\eta_{\mu}\right|>1.2$

- c) one muon is detected in the barrel, the other in the endcaps.

Table 5 shows the contributions of the quarkonia and background in these 3 regions as well as the S/B ratio. This table shows different trends according to whether the $J / \psi$ 's or the $\Upsilon$ 's samples are studied.

Because of the relatively low rest mass of the $J / \psi$, muons need to have a high $p_{\mathrm{T}}$ to be detected in the barrel region. This fact was already mentioned (Sect. 4.2). The consequence is that more than $75 \%$ of the detected $J / \psi$ are in the encaps where the $\mathrm{S} / \mathrm{B}$ is the lowest. Selecting muons in the barrel will obviously give a cleaner spectrum but with a drastically lower statistics for the $J / \psi$.

The situation is quite different for the $\Upsilon$. The table indicates that detecting both muons in the barrel region or in the endcaps will lead to higher $\mathrm{S} / \mathrm{B}$ values than the value $\mathrm{S} / \mathrm{B}=0.07$ obtained without doing this selection. Taking into account the "mixed region" increases very much the number of background events while the number of $\Upsilon$ is approximately the same than in the other cases. In this "mixed region", the S/B ratio is lower by one order of magnitude. Rejecting dimuons from this region gives a ratio $\mathrm{S} / \mathrm{B}=0.20,3$ times higher than the value $\mathrm{S} / \mathrm{B}=0.07$ got without the selection.

Table 5: The expected number of quarkonia and background events obtained in $10^{5}$ high multiplicity $\left(d N^{ \pm} / d \eta=\right.$ 5000) $\mathrm{Pb}+\mathrm{Pb}$ collisions in 3 detection regions.

\begin{tabular}{|rr|r|r|r|}
\cline { 3 - 5 } \multicolumn{1}{c|}{} & quarkonia & background & S/B \\
\hline & $\eta_{\mu_{1}}<1.2$ and $\eta_{\mu_{2}}<1.2$ & 0.0458 & 0.0159 & 2.8772 \\
& $\eta_{\mu_{1}}>1.2$ and $\eta_{\mu_{2}}>1.2$ & 0.3256 & 0.6668 & 0.4883 \\
& $\eta_{\mu_{1}}<1.2$ and $\eta_{\mu_{2}}>1.2$ & 0.0475 & 0.0482 & 0.9856 \\
\hline & $\eta_{\mu_{1}}<1.2$ and $\eta_{\mu_{2}}<1.2$ & 0.0234 & 0.0939 & 0.2482 \\
$\Upsilon$ & $\eta_{\mu_{1}}>1.2$ and $\eta_{\mu_{2}}>1.2$ & 0.0156 & 0.0984 & 0.1582 \\
& $\eta_{\mu_{1}}<1.2$ and $\eta_{\mu_{2}}>1.2$ & 0.0176 & 0.6590 & 0.0268 \\
\hline
\end{tabular}

\subsection{The reconstruction errors in dimuon mass spectrum}

Systematic errors due to limited statistics have been estimated for the spectra obtained with fast Monte Carlo generation. These are the errors of the weighting method. These errors can be easily calculated and can be summed with statistical errors in square. However, one has to mention that errors on the multiplicity dependence are not included. Taking into account that most of pairs are in the endcap region, the errors due to multiplicity dependence are at the level of a few percents (Fig.10). No other sources of systematics is discussed in this section.

The L1 condition for dimuon detection in heavy ion collisions is to have at least one muon candidate. The low quality muon candidates are used in L1 to improve the trigger efficiency of $\mathrm{J} / \psi$ [22]. The dimuon candidate pair is selected at High Level Trigger (HLT) with combination of either two muons at L1 or two muons at L2. Each of the particle in the pair has the probability to be registered by L1 trigger $\left(w_{i}^{L 1}, w_{j}^{L 1}\right)$ and(or) by L2 $\operatorname{trigger}\left(w_{i}^{L 2}, w_{j}^{L 2}\right)$. Sometimes, particle can have L1 muon candidate, but trigger conditions are failed for L2 muon candidate and vice-verse particle can have only muon trigger candidate at L2.

The high level trigger and reconstruction weight applied to each pair of particles is:

$$
W_{i j}=W_{i j}^{t r i g} \times W_{i j}^{r e c},
$$

where $W_{i j}^{\text {trig }}$ is the efficiency to trigger the pair of the particles $i$ and $j$ at HLT and $W_{i j}^{r e c}$ is the reconstruction efficiency of the pair. 
The variance in dimuon mass spectrum is:

$$
D\left(W_{i j}\right)=\left(W_{i j}^{r e c}\right)^{2} \times D\left(W_{i j}^{\text {trig }}\right)+\left(W_{i j}^{\text {trig }}\right)^{2} \times D\left(W_{i j}^{r e c}\right)
$$

The HLT weight of the pair of particles with combination of either two muons at L1 or two muons at L2 is:

$$
\begin{gathered}
W_{i j}^{\text {trig }}=w_{i}^{L 1} * w_{i}^{L 2} * w_{j}^{L 1} * w_{j}^{L 2}+ \\
w_{i}^{L 1} *\left(1-w_{i}^{L 2}\right) * w_{j}^{L 1} * w_{j}^{L 2}+ \\
w_{i}^{L 1} * w_{i}^{L 2} * w_{j}^{L 1} *\left(1-w_{j}^{L 2}\right)+ \\
\left(1-w_{i}^{L 1}\right) * w_{i}^{L 2} * w_{j}^{L 1} * w_{j}^{L 2}+ \\
w_{i}^{L 1} * w_{i}^{L 2} *\left(1-w_{j}^{L 1}\right) * w_{j}^{L 2}+ \\
\left(1-w_{i}^{L 1}\right) * w_{i}^{L 2} *\left(1-w_{j}^{L 1}\right) * w_{j}^{L 2}+ \\
w_{i}^{L 1} *\left(1-w_{i}^{L 2}\right) * w_{j}^{L 1} *\left(1-w_{j}^{L 2}\right)
\end{gathered}
$$

The probability to be registered by HLT is the sum of the probabilities, following terms in the formula:

- each particle of the pair has both L1 and L2 trigger candidates

- first particle has only L1 trigger candidate

- second particle has only L1 trigger candidate

- first particle has only L2 trigger candidate

- second particle has only L2 trigger candidate

- both particles have only L2 trigger candidates

- both particles have only L1 trigger candidates

The variance is:

$$
\begin{gathered}
D\left(W_{i j}^{\text {trig }}\right)=7 \times\left(\left(w_{i}^{L 1} * w_{i}^{L 2} * w_{j}^{L 1}\right)^{2} * D\left(w_{j}^{L 2}\right)+\right. \\
\left(w_{i}^{L 1} * w_{i}^{L 2} * w_{j}^{L 2}\right)^{2} * D\left(w_{j}^{L 1}\right)+ \\
\left(w_{i}^{L 1} * w_{j}^{L 1} * w_{j}^{L 2}\right)^{2} * D\left(w_{i}^{L 2}\right)+ \\
\left.\left(w_{i}^{L 2} * w_{j}^{L 1} * w_{j}^{L 2}\right)^{2} * D\left(w_{i}^{L 1}\right)\right)
\end{gathered}
$$

The reconstruction efficiency is:

$$
W_{i j}^{r e c}=w_{i}^{r e c} \times w_{j}^{r e c}
$$

The variance is:

$$
D\left(W_{i j}^{r e c}\right)=\left(w_{i}^{r e c}\right)^{2} * D\left(w_{j}^{r e c}\right)+\left(w_{j}^{r e c}\right)^{2} * D\left(w_{i}^{r e c}\right)
$$

Finally, the error in the mass spectrum due to limited statistics of the trigger and reconstruction tables is:

$$
\operatorname{Err}(M)=\sqrt{\sum_{i} \sum_{j} D\left(W_{i j}\right)}
$$

Figures 35-38 presents the dimuon mass spectra for the low and high multiplicity assumptions with the error band calculated with 9 . 


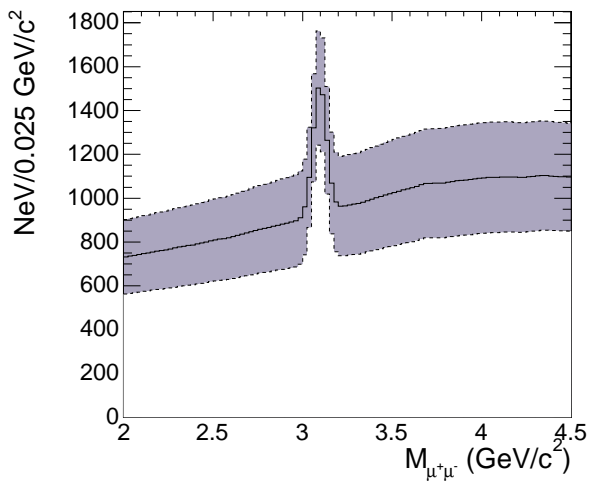

Figure 35: Opposite-sign dimuon mass distribution obtained for the high multiplicity assumption in the $J / \psi$ mass range

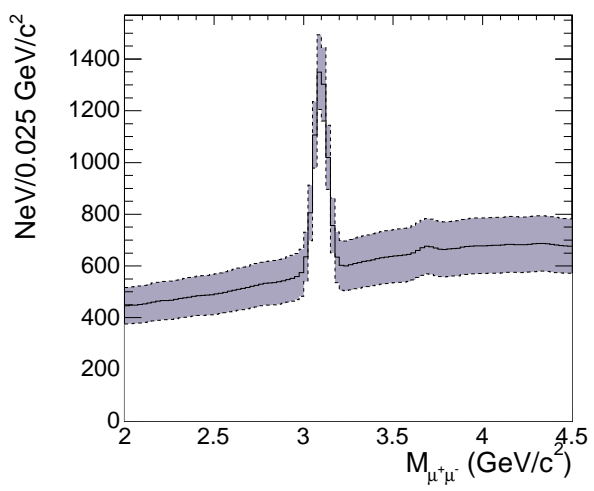

Figure 37: Opposite-sign dimuon mass distribution obtained for the low multiplicity assumption in the $J / \psi$ mass range

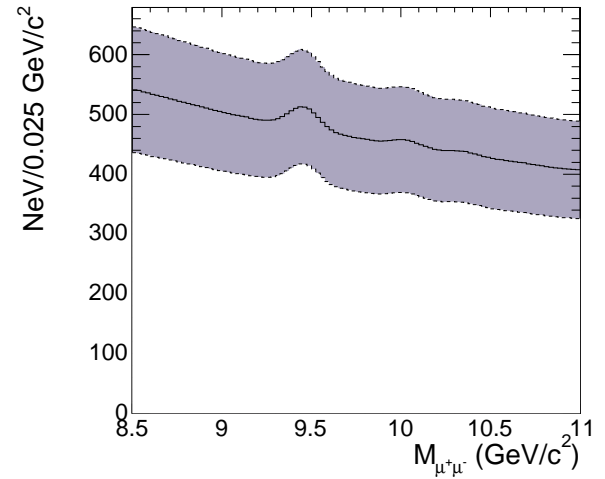

Figure 36: Opposite-sign dimuon mass distribution obtained for the high multiplicity assumption in the $\Upsilon$ mass range

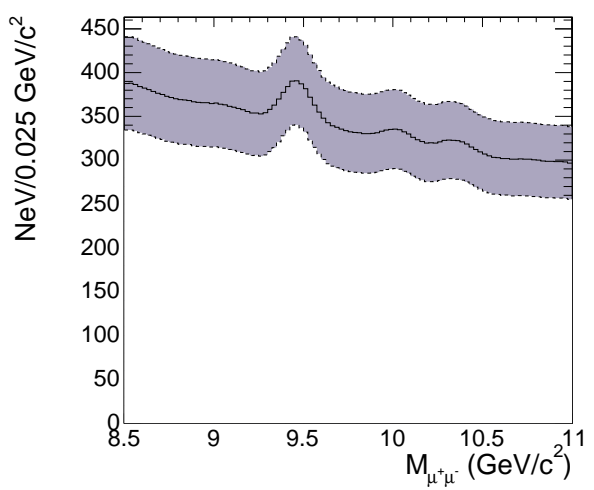

Figure 38: Opposite-sign dimuon mass distribution obtained for the low multiplicity assumption in the $\Upsilon$ mass range

The error, calculated with 9 amounts to $20 \%$ at the $J / \psi$ and $25 \%$ at the $\Upsilon$.

\subsection{Comparison of the Fast Monte Carlo and the detailed simulation}

\subsubsection{Reconstruction of HIJING events}

$500 \mathrm{~K}$ events of $\mathrm{PbPb}$ events were generated with HIJING with quenching switched off [6]. Since HIJING does not allow simultaneous production of $c \bar{c}$ and $b \bar{b}$ pairs these last contribution ( $b \bar{b}$ pairs) have been superimposed to the HIJING events before the full simulation and the analysis. The production of $c \bar{c}$ pairs is taken as in HIJING default. The production of $b \bar{b}$ pairs was done according to [16]. The cross-section of $b \bar{b}\left(\sigma_{b \bar{b}}\right)$ is taken to be 0.220 $\mathrm{mb}$. The number of $b \bar{b}$ pairs in the event at the fixed impact parameter $b$ is randomize with Poissonian distribution and

$$
<N_{b \bar{b}}>(b)=T(b) * \sigma_{b \bar{b}}
$$

, where $\mathrm{T}(\mathrm{b})$ is the nuclear overlap function. A 5-order polynomial is taken to parametrize $\mathrm{T}(b)$ :

$$
\begin{gathered}
T(b)=30.401+0.2746 * b-0.95902 * b * * 2+0.99466 E-01 * b * * 3 \\
-0.38302 * b * * 4+0.51598 E-04 * b * * 5
\end{gathered}
$$

The $500000 \mathrm{~Pb}-\mathrm{Pb}$ events were passed through L1 and L2 muon trigger with condition: either two opposite sign muons at L1 or two opposite sign muons at L2 (Sect.3.3.2). About 8000 events were selected by the L1/L2 trigger condition. These events were reconstructed in tracker with L3 reconstruction logic (Sect 3.3.2). About 2500 events were survived after reconstruction in the tracker. The overall high level trigger efficiency for HIJING event with $b \bar{b}$ 
production is 0.005 . One has to mention, that these are all background "dimuons". The cross-section for $J / \psi$ (in HIJING the cross-section for $c \bar{c}$ production is 100 times less than the one used in the current simulations based on the NLO calculations) and $\Upsilon$ are too low to have more then a few tens of resonances in the HIJING sample in the full phase space. Taking into account the acceptance, only a few resonaces may appear in the dimuon mass spectra produced in the detailed simulation and reconstruction of 500 Kevents with HIJING.

Figure 39 shows the $\eta_{\mu_{1}}$ vs $\eta_{\mu_{2}}$ distribution for dimuons with mass more than $12 \mathrm{GeV} / \mathrm{c}^{2}$ and figure 40 shows the same distribution for the dimuon mass less than $12 \mathrm{GeV} / \mathrm{c}^{2}$. The reconstruction of the HIJING events was done with L3 trigger reconstruction logic (only one pair is reconstructed in the event) while the fast Monte-Carlo program uses off-line reconstruction logic (all pairs corresponding to the L1/L2 dimuon candidates are reconstructed). The fake pair masses are distributed in a wide mass interval from 0 to $20 \mathrm{GeV}$. The dimuon mass spectrum obtained from the detailed simulation was multiplied to the factor 1.1 to compensate for the average number of the additional combinatorial pairs found in the offline reconstruction. The additional muons are mainly concentrated in the pseudorapidity range $2.1<|\eta|<2.4$ and thus affect more the $J / \psi$ mass range.

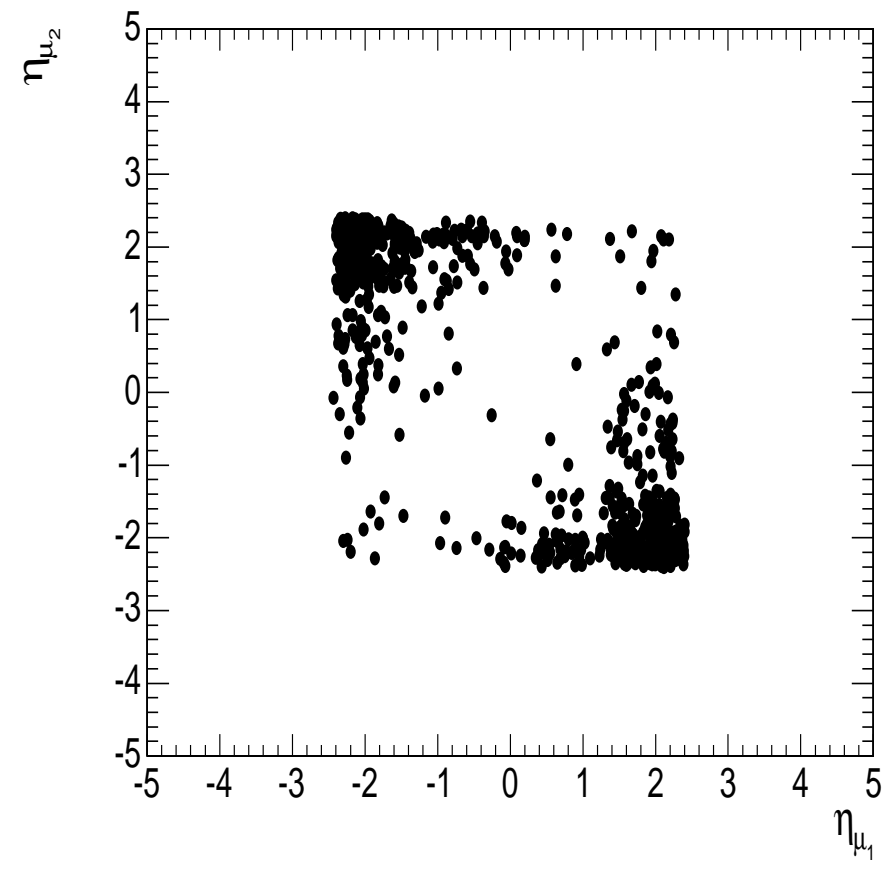

Figure 39: The distribution of $\mid \eta_{\mu_{1}}$ vs $\eta_{\mu_{2}} \mid$ for pairs with dimuon mass more then $12 \mathrm{GeV} / \mathrm{c}^{2}$ 


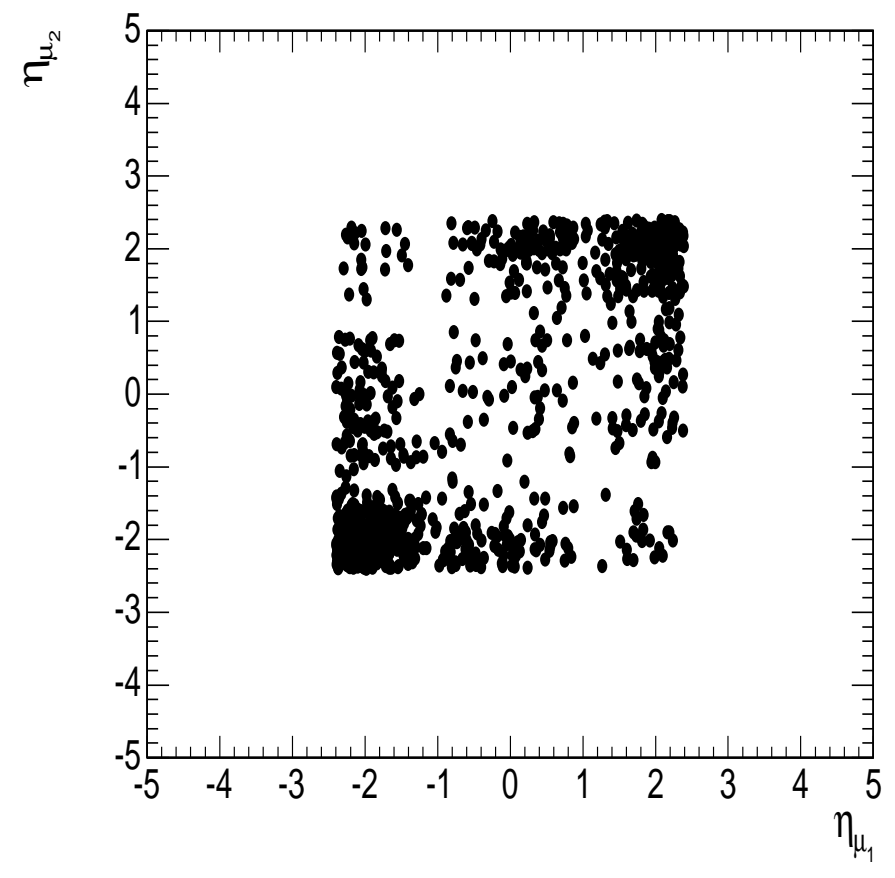

Figure 40: The distribution of $\mid \eta_{\mu_{1}}$ vs $\eta_{\mu_{2}} \mid$ for pairs with dimuon mass less then $12 \mathrm{GeV} / \mathrm{c}^{2}$

\subsubsection{Comparison of fast Monte Carlo with detailed simulations}

For the fast MC the kinematical distributions correspond to those used in the detailed simulation of HIJING events:

- the multiplicity of pions and kaons is at the level of $91 \%$ of the HIJING charged particle multiplicity,

- multiplicity and spectra of the muons from $c \bar{c}$ production come from the HIJING event generator

- multiplicity and spectra of the muons from $b \bar{b}$ production are produced according to [16]

Figure 41 presents the comparison of the $500 \mathrm{~K}$ events simulated with fast monte-carlo method and $500 \mathrm{~K}$ events done with detailed simulation. The results of the detailed simulation are multiplied to a factor 1.1 to compensate the difference between level three and offline logic in the muon track reconstruction. The error bars shown for the fast Monte Carlo results include both statistical and systematic (Sect.5.4) errors. The fast Monte Carlo spectrum is smoother then the detailed simulation spectrum. The reasons are in simplification of the approach used in the fast Monte-Carlo. The mass resolution is taken constant at $85 \mathrm{MeV} / \mathrm{c}^{2}$ for the range from $5 \mathrm{GeV} / \mathrm{c}^{2}$ to $40 \mathrm{GeV} / \mathrm{c}^{2}$. The mass resolution in the detailed simulation is roughly $0.009 \times \mathrm{M}$, a little bit higher in the low mass region and lower in the high mass region. Moreover, the coarse bins (only four bins in $\eta$ and four bins in momentum) were used to determine the dependence of the reconstruction efficiency on $\eta$ and momentum. The multiplicity dependence of the efficiency was also analyzed in the coarse bins in $\eta$ and was integrated over the momentum. A more tiny division requires large amount of the calculation time.

Resonances $J / \psi$ and $\Upsilon$ are not added in the fast monte carlo as they are almost absent in the detailed simulation. Only background events in the fast Monte Carlo simulation and detailed simulation are compared. 


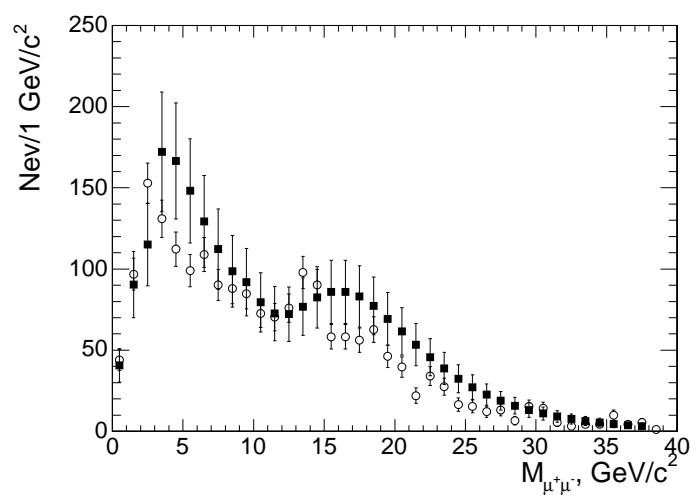

Figure 41: Comparison of the fast MC result (full squares) with a full HIJING simulation (empty circles). The mass resolution in the fast Monte Carlo is taken constant at $85 \mathrm{MeV} / \mathrm{c}^{2}$

The mass resolution in the fast Monte Carlo is taken as $0.009 \times \mathrm{M}$ in Fig. 42 .

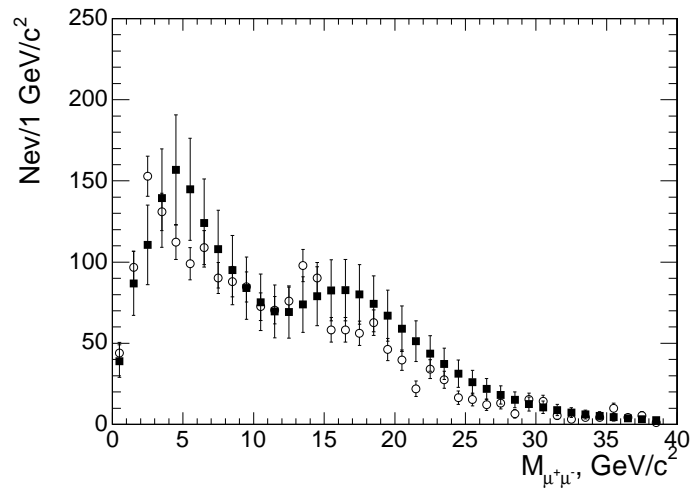

Figure 42: Comparison of the fast MC result (full squares) with a full HIJING simulation (empty circles). The mass resolution in the fast Monte Carlo is taken as $0.009 \times \mathrm{M}$

The agreement between both simulation is excellent, less than $10 \%$ discrepancy, much smaller than the systematics uncertainties due to the limited statistics in the trigger and reconstruction tables (Sect 5.4). This comparison shows that the fast MC simulation gives believable predictions on the detector response.

\section{Discussion on the results and conclusions}

With its $\approx 4 \pi$ muon acceptance and full calorimetric coverage, CMS can make very significant and, in some respects, unique contribution to heavy ion physics. Studies of the $\Upsilon$ family, from $p p$ to $\mathrm{Pb}+\mathrm{Pb}$, as well as from peripheral to central collisions, is likely to be of great interest at the LHC, just as the $J / \psi$ has been for the SPS.

The key issue for CMS is the muon reconstruction efficiency in the tracker under conditions of extreme occupancies expected in $\mathrm{Pb}+\mathrm{Pb}$ collisions. The reconstruction efficiency is in the range from $90 \%$ to $84 \%$ for dimuons with both muons in $|\eta|<0.8$ for event multiplicity up to $d N_{\mathrm{ch}} / d \eta=5000$. For muon pairs with at least one muon in the range $0.8<|\eta|<2.4$ the efficiency is $70 \%$ for multiplicity up to $d N_{\mathrm{ch}} / d \eta=2500$ and falls down to $40 \%$ for multiplicities above 2500 per unit of pseudorapidity. The purity is kept above $80 \%$. The $\Upsilon$ mass resolution is $56 \mathrm{MeV}$ for the dimuons with both muons within pseudorapidity range $|\eta|<0.8$. For the entire pseudorapidity region $|\eta|<2.4$, the $\Upsilon$ mass resolution is $85 \mathrm{MeV}$ while the $J / \psi$ mass resolution is $35 \mathrm{MeV}$.

A fast Monte Carlo program was developed on the basis of the trigger and reconstruction efficiency tables created for the different particle species. The fast Monte Carlo approach has been validated via a comparison with the detailed simulation based on the same generator input.

The dimuon mass spectra are obtained with the fast Monte-Carlo program for the min bias $\mathrm{Pb}-\mathrm{Pb}$ events. 
The large rapidity aperture of the muon detector, as well as the precise tracking, result in high statistics and a very good separation between the $\Upsilon$ states. The higher masses of the $\Upsilon$ states favour their measurement in the barrel where the muon $p_{\mathrm{T}}$ detection threshold is limited to $3.5 \mathrm{GeV} / c$ whereas the $J / \psi$ needs $p_{\mathrm{T}}>6 \mathrm{GeV} / c$. Assuming only cold matter effect of shadowing, the number of events (20000 or 25000 for $\Upsilon$ depending on the multiplicity set and 140000 or 170000 for $\mathrm{J} / \psi$ ) for one month of data taking $\left(0.5 \mathrm{nb}^{-1}\right)$ is high enough to provide a comparison between the several impact parameter bins and to carry out the study of the more differential analysis $(d N / d y$, $\left.d N / d p_{T}\right)$.

\section{Acknowledgments}

The authors wish to express their gratitude to D. Acosta, D. d' Enterria and L. Foa for the fruitful comments and discussions. Special acknowlegment is adressed to R. Vogt for the valuable discussions and theoretical support. The authors wish to express their gratituge to J.Lee, M.Balateen and I.Loktin for help with simulation. 


\section{References}

[1] M. C. Abreu et al. [NA50 Collaboration], Phys. Lett. B450 (1999) 456.

[2] M. C. Abreu et al. [NA50 Collaboration], Phys. Lett. B477 (2000) 28.

[3] H. Periera [PHENIX Collaboration] in proceedings of Quark Matter'05, Budapest, Hungary,August 2005.

[4] R. Vogt, nucl-th/0507027.

[5] Karsch, Kharzeev and Satz, BNL-NT-05/50.

[6] M.Gyulassy and X.-N. Wang, Phys.Rev D44(1991) 3501

[7] OSCAR user's Guide at www.http://cmsdoc.cern.ch/swsi.html

[8] ORCA user's Guide at www.http://cmsdoc.cern.ch/swsi.html

[9] HCAL Technical Design Report, CERN/LHCC 97-31, 1997.

[10] MUON Technical Design Report, CERN/LHCC 97-32, 1997.

[11] ECAL Technical Design Report,CERN/LHCC 97-33, 1997.

[12] Tracker Technical Design Report,CERN/LHCC 98-6, 1998.

[13] DAQ TDR CERN/LHCC/2002-26

[14] L.Grandchamp and R.Rapp, Nucl.Phys. A715, 545(2003), hep-ph/0209141.

[15] Torbjrn Sjstrand, Leif Lnnblad, Stephen Mrenna, PYTHIA 6.2 Physics and Manual, hep-ph/0108264.

[16] M. Bedjidian et al, Hard Probes in Heavy-Ion Collisions at the LHC, CERN-2004-009, hep-ph/0311048.

[17] M. C. Abreu et al. [NA50 Collaboration] Phys. Lett. B410 (1997) 327.

[18] V.D. Barger, W.Y. Keung and R.J. Phillips, Phys. Lett. B91 (1980) 253.

[19] V.D. Barger, W.Y. Keung and R.J. Phillips, Z. Phys. C6 (1980) 169.

[20] D. Kharzeev, E. Levin and M. Nardi, arXiv:hep-ph/0111315.

[21] D.Chrisman,T.Moers, CMS TN/93-106.

[22] CMS Collaboration, The Physics Technical Design Report, part I, CERN-LHCC-2006-001

[23] ALICE Physics Performance Report, vol. II, CERN/LHCC 2005-030.

[24] M.Bedjidian, O.Kodolova, S.Petrushanko, CMS NOTE 1999/004.

[25] B.I.Abelev at al, nucl-ex/0606003 v3. 